\title{
Single-Molecule Charge Transport Through Positively Charged Electrostatic Anchors
}

Hongliang Chen ${ }^{1}$, Vitor Brasiliense ${ }^{1,5}$, Jingshan Mo, ${ }^{6}$ Long Zhang ${ }^{1}$, Yang Jiao ${ }^{1}$, Zhu Chen ${ }^{1}$, Leighton O. Jones ${ }^{1}$, Gen He, ${ }^{6}$ Qing-Hui Guo ${ }^{1}$, Xiao-Yang Chen ${ }^{1}$, Bo Song ${ }^{1}$, George C. Schatz ${ }^{1}$, and J. Fraser Stoddart $1,2,3,4 *$

${ }^{1}$ Department of Chemistry, Northwestern University, 2145 Sheridan Road, Evanston, Illinois 60208, USA

${ }^{2}$ School of Chemistry, University of New South Wales, Sydney, NSW 2052, Australia

${ }^{3}$ Stoddart Institute of Molecular Science, Department of Chemistry, Zhejiang University,

Hangzhou 310021, China

${ }^{4}$ ZJU-Hangzhou Global Scientific and Technological Innovation Center, Hangzhou 311215 ,

China

${ }^{5}$ Université Paris-Saclay, ENS Paris-Saclay, CNRS, PPSM, 4 avenue des Sciences, 91190 Gif/Yvette, France

${ }^{6}$ State Key Laboratory of Optoelectronic Materials and Technologies, School of Electronics and Information Technology, Sun Yat-Sen University, Guangzhou 510006, China

* Email: stoddart@,northwestern.edu (J.F.S.)

\section{Supporting Information}

\section{Table of Contents}

Section A. Materials / General Methods / Instruments ......... S2

Section B. Synthetic Protocols .............................................. S3

Section C. NMR Spectroscopy ........................................... S9

Section D. Supplemental Characterizations ........................ S19

Section E. Calculation Methods ......................................... S22

Section F. Supplemental Electrical Measurements ............ S26

Section G. Supplemental References................................... S33 


\section{Section A. Materials / General methods / Instruments}

All reagents were purchased from commercial suppliers and employed without further purification unless stated otherwise. $\mathrm{MeCN}$ for reactions was prepared using a solvent drying system. Anhydrous MeCN for electrolysis and UV-Vis were purchased from Aldrich (anhydrous, 99.8\%). Microwave-assisted reactions were performed on Biotage Microwave Initiator 2.5 with automatic sample loader. Thin layer chromatography (TLC) was performed on silica gel 60 F254 (E. Merck). Column chromatography, including both normal phase (RediSep Rf Gold ${ }^{\circledR}$ Normal-Phase Silica) and reversed-phase (RediSep Rf Gold ${ }^{\circledR}$ Reversed-Phase C18), were carried out using CombiFlash ${ }^{\circledR}$ Automation Systems (Teledyne ISCO). UV-Vis Absorption spectra were recorded in a conventional rectangular quartz cuvette (4 mm optical path) on a UV-3600 Shimadzu spectrophotometer at room temperature. Routine nuclear magnetic resonance (NMR) spectra were recorded on Bruker Avance 500 spectrometers, with working frequencies of $500 \mathrm{MHz}$ for ${ }^{1} \mathrm{H}$, and $125 \mathrm{MHz}$ for ${ }^{13} \mathrm{C}$ nuclei, respectively. Chemical shifts are reported in ppm relative to the signals corresponding to the residual nondeuterated solvents $\left(\mathrm{CD}_{3} \mathrm{CN}: \delta_{\mathrm{H}}=1.94 \mathrm{ppm}\right.$ and $\delta_{\mathrm{C}}=1.32$ and $118.26 \mathrm{ppm}$. X-Ray photoelectron spectroscopy (XPS) characterizations were carried out on Thermo ESCALab $250 \mathrm{Xi}$, (AlK-alpha X-ray radiation, $1486.6 \mathrm{eV}$ ). XPS depth analysis was performed by sputtering Ar ion on to the BIPY-Me•2PF 6 film. The BIPY and BIPY-Me $2 \mathrm{PF}_{6}$ monolayer films were prepared by spin-coating. The thickness and coverage can be turned ${ }^{1}$ by changing the rotating speed from 3000 to $6000 \mathrm{rpm}$. 


\section{Section B. Synthetic Protocols}

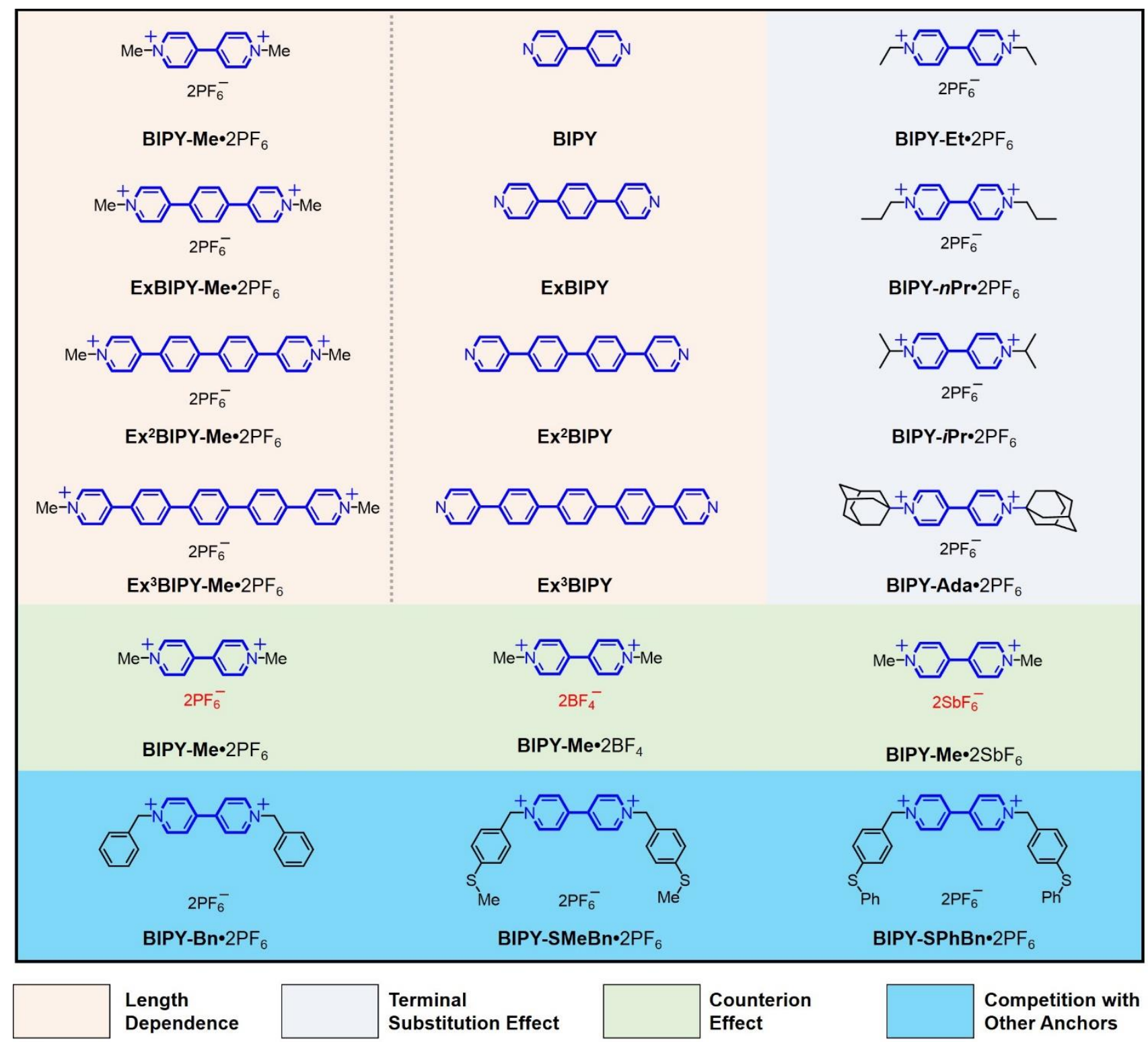

Scheme S1. Structural formulas and descriptors for compounds 


\section{(1)Synthesis of Dimethyl Extend Viologens}

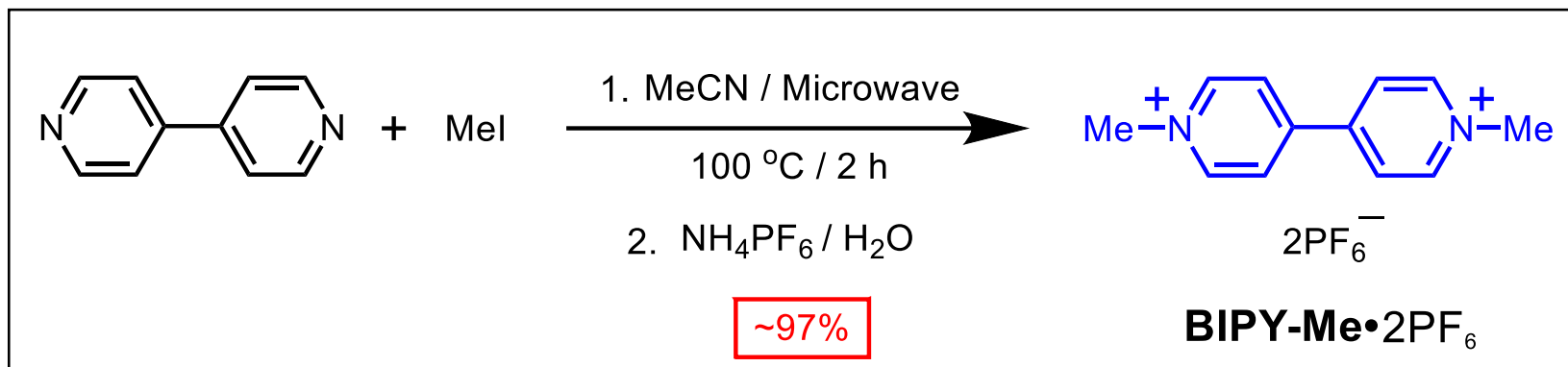

Scheme S2. Synthesis of BIPY-Me•2PF 6 .

BIPY-Me•2PF6: An MeCN (5 mL) solution of 4,4'-bipyridine (100 mg) and excess of MeI ( 10 equiv) were introduced into a microwave vial. The reaction mixture was irradiated in a microwave apparatus at $100{ }^{\circ} \mathrm{C}, 50 \mathrm{~W}$ for $2 \mathrm{~h}$. After the reaction mixture had cooled to ambient temperature, the precipitate was filtered off and the unreacted $\mathrm{MeI}$ was removed with multiple $\mathrm{Et}_{2} \mathrm{O}$ washes to obtain the pure BIPY-Me•2I. Finally, BIPY-Me•2I was dissolved in $\mathrm{H}_{2} \mathrm{O}(\sim 20 \mathrm{~mL})$ and BIPY$\mathrm{Me} \cdot 2 \mathrm{PF}_{6}$ was reprecipitated by adding solid $\mathrm{NH}_{4} \mathrm{PF}_{6}$ salt, and collected by filtration. Excess of $\mathrm{NH}_{4} \mathrm{PF}_{6}$ was removed by washing several times with $\mathrm{MeOH}$ and $\mathrm{Et}_{2} \mathrm{O}$ to obtain the pure white solid. in $\sim 97 \%$ yield. ${ }^{1} \mathrm{H}$ NMR (500 MHz, $\left.\mathrm{CD}_{3} \mathrm{CN}, \mathrm{ppm}\right): \delta=8.84(\mathrm{~d}, J=6.7 \mathrm{~Hz}, 4 \mathrm{H}), 8.37$ (d, $J$ $=6.1 \mathrm{~Hz}, 4 \mathrm{H}), 4.40(\mathrm{~s}, 6 \mathrm{H}) .{ }^{13} \mathrm{C} \mathrm{NMR}\left(125 \mathrm{MHz}, \mathrm{CD}_{3} \mathrm{CN}, \mathrm{ppm}\right): \delta=150.5,147.4,127.7,49.5$.

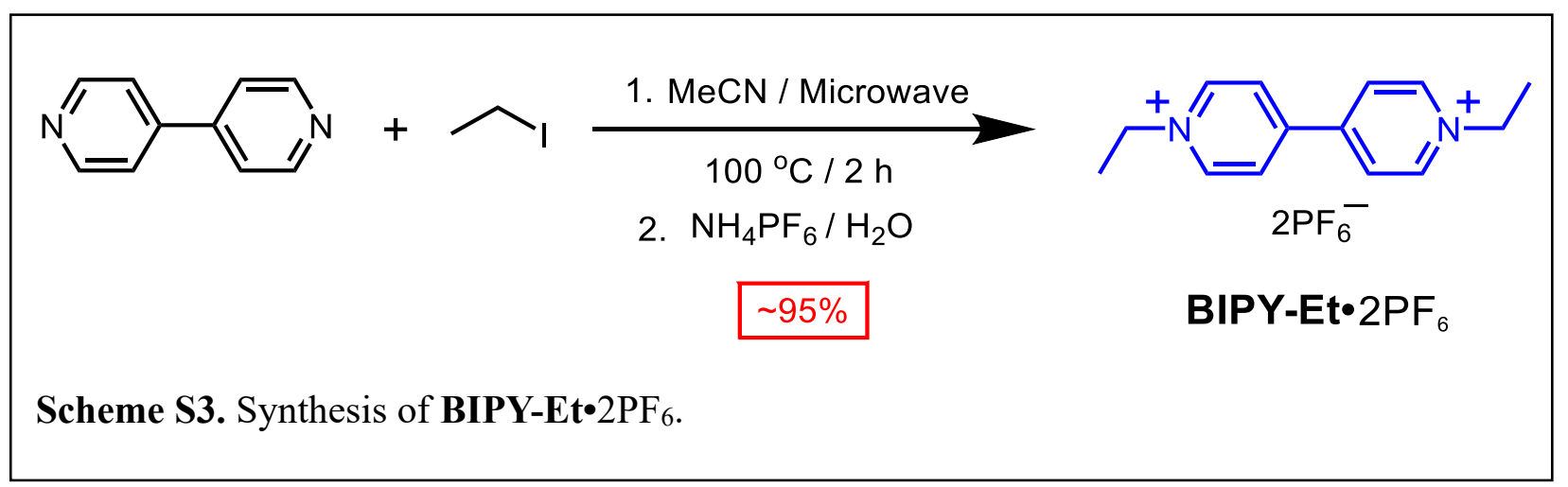

BIPY-Et-2PF6: This compound was prepared using the same methodology as used in the preparation of BIPY-Me $2 \mathrm{PF}_{6} \cdot \mathbf{B I P Y}-\mathbf{E t} \cdot 2 \mathrm{PF}_{6}$ was obtained as a white solid with a yield in $\sim 95 \%$. ${ }^{1} \mathrm{H}$ NMR (500 MHz, CD 3 CN, ppm): $\delta=8.91(\mathrm{~d}, J=6.6 \mathrm{~Hz}, 4 \mathrm{H}), 8.38(\mathrm{~d}, J=5.9 \mathrm{~Hz}, 4 \mathrm{H}), 4.68(\mathrm{q}$, 
$J=7.3 \mathrm{~Hz}, 4 \mathrm{H}), 1.66(\mathrm{t}, J=7.3 \mathrm{~Hz}, 6 \mathrm{H}) .{ }^{13} \mathrm{C}$ NMR $\left(125 \mathrm{MHz}, \mathrm{CD}_{3} \mathrm{CN}, \mathrm{ppm}\right): \delta=150.8,146.3$, 128.1, 58.7, 16.6.

\section{(2) Synthesis of Single-Channel Compounds}

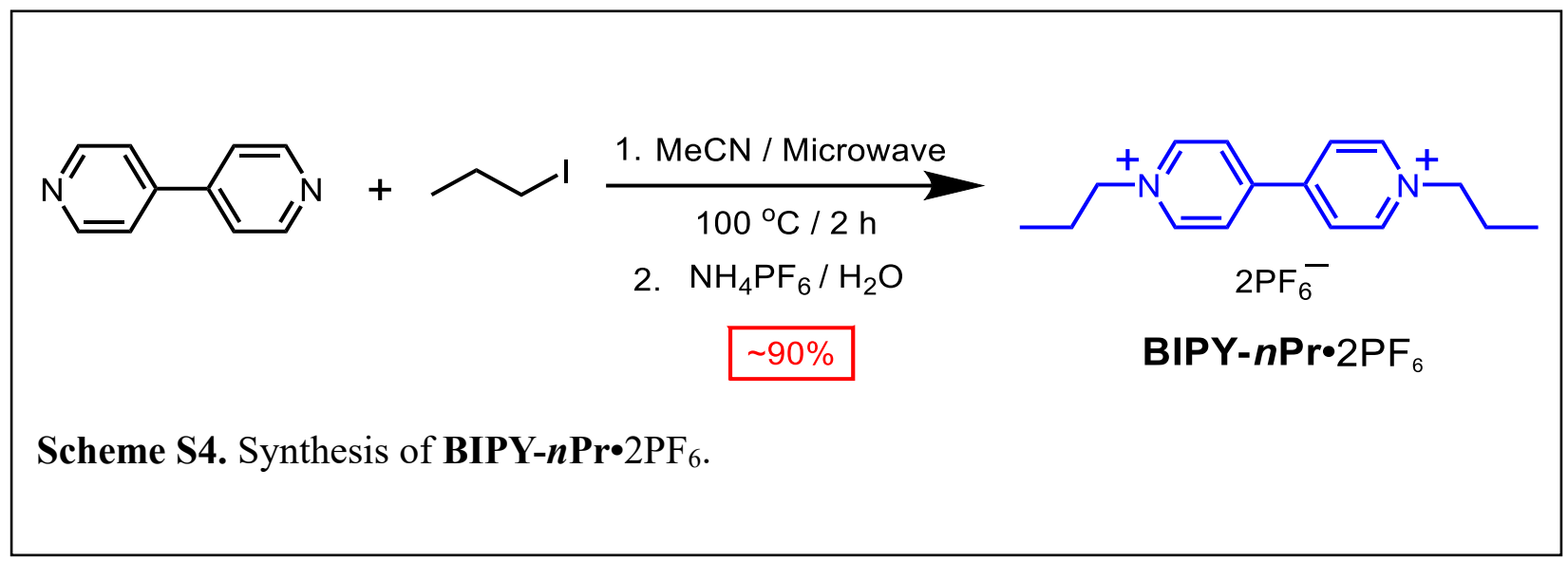

BIPY-nPr-2PF6: This compound was prepared using the same methodology as used in the preparation of BIPY-Me $2 \mathrm{PF}_{6} . \mathbf{B I P Y}-\boldsymbol{n P r} \cdot 2 \mathrm{PF}_{6}$ was obtained as a white solid with a yield in $\sim 90 \%$. ${ }^{1} \mathrm{H}$ NMR (500 MHz, CD $\left.{ }_{3} \mathrm{CN}, \mathrm{ppm}\right): \delta=8.89(\mathrm{~d}, J=6.9 \mathrm{~Hz}, 4 \mathrm{H}), 8.38(\mathrm{~d}, J=6.4 \mathrm{~Hz}, 4 \mathrm{H}), 4.59$ (q, $J=7.4 \mathrm{~Hz}, 4 \mathrm{H}), 2.06(\mathrm{~h}, J=7.4 \mathrm{~Hz}, 4 \mathrm{H}), 1.00(\mathrm{t}, J=7.3 \mathrm{~Hz}, 6 \mathrm{H}) \cdot{ }^{13} \mathrm{C} \mathrm{NMR}\left(125 \mathrm{MHz}, \mathrm{CD}_{3} \mathrm{CN}\right.$, ppm): $\delta=150.9,146.5,128.1,64.4,25.4,10.5$.

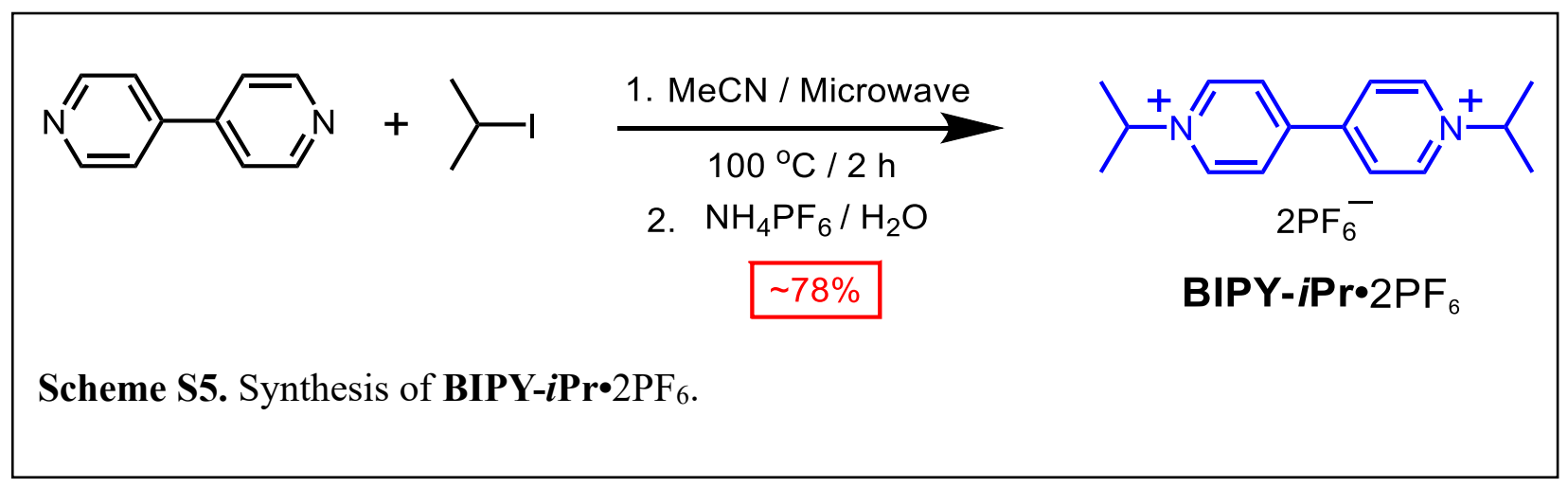

BIPY-iPr•2PF6: This compound was prepared using the same methodology as used in the preparation of $\mathbf{B I P Y}-\mathbf{M e} \cdot 2 \mathrm{PF}_{6}$. BIPY- $\boldsymbol{n} \mathbf{P r} \cdot 2 \mathrm{PF}_{6}$ was obtained as a white solid with a yield in $\sim 78 \%$. ${ }^{1} \mathrm{H}$ NMR (500 MHz, CD $\left.3 \mathrm{CN}, \mathrm{ppm}\right): \delta=8.98$ (d, $\left.J=7.0 \mathrm{~Hz}, 4 \mathrm{H}\right), 8.39$ (d, $\left.J=5.8 \mathrm{~Hz}, 4 \mathrm{H}\right), 5.03$ (hept, $J=6.7 \mathrm{~Hz}, 2 \mathrm{H}), 1.70$ (d, $J=6.4 \mathrm{~Hz}, 12 \mathrm{H}) .{ }^{13} \mathrm{C} \mathrm{NMR}\left(125 \mathrm{MHz}, \mathrm{CD}_{3} \mathrm{CN}, \mathrm{ppm}\right): \delta=150.6$, $144.5,127.8,66.4,22.6$. 

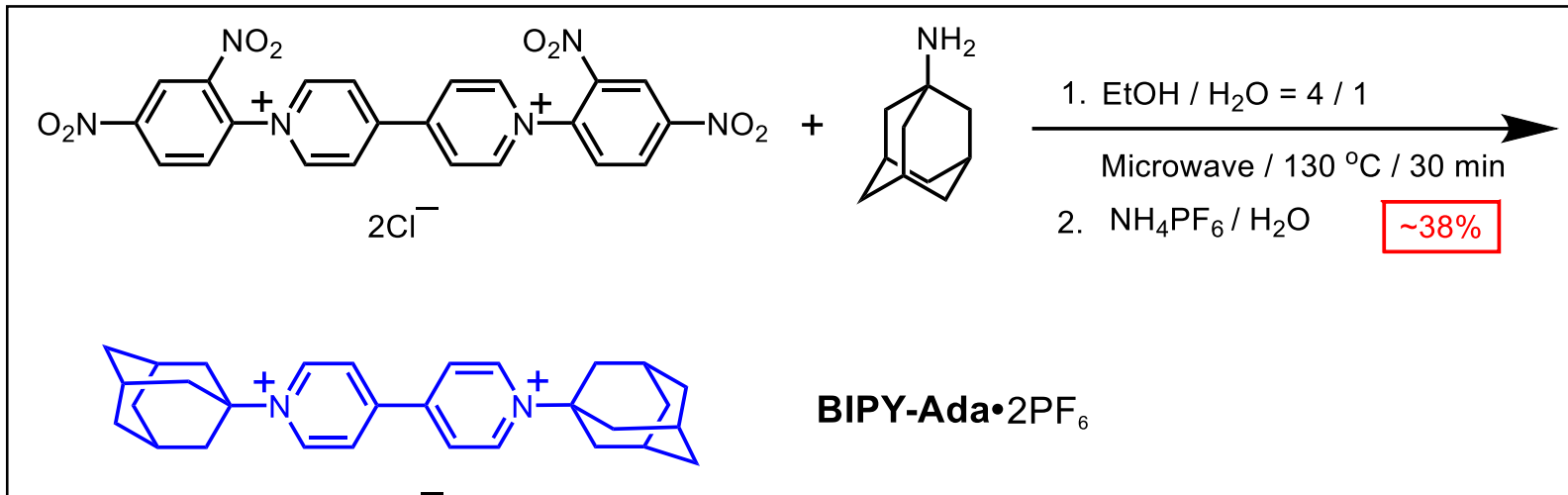

BIPY-Ada·2PF

$2 \mathrm{PF}_{6}^{-}$

Scheme S6. Synthesis of BIPY-Ada・ $2 \mathrm{PF}_{6}$.

BIPY-Ada-2PF6: 1,1'-Bis(2,4-dinitrophenyl)-4,4'-bipyridinium dichloride (100 mg) and 1-adamantylamine $(80 \mathrm{mg})$ were introduced into a microwave vial and dissolved in a 80:20 (v/v) $\mathrm{EtOH} / \mathrm{H}_{2} \mathrm{O}$ mixture $(2 \mathrm{ml})$. The reaction mixture was irradiated in a microwave apparatus at 130 ${ }^{\circ} \mathrm{C}, 50 \mathrm{~W}$ for $30 \mathrm{~min}$. After the reaction mixture had cooled to ambient temperature, the solvent was removed in vacuo, and the dark brown residue was stirred in $\mathrm{Et}_{2} \mathrm{O}(100 \mathrm{~mL})$ at room temperature for $30 \mathrm{~min}$ and then filtered. The solid was dissolved in $\mathrm{H}_{2} \mathrm{O}(100 \mathrm{~mL})$ and then filtered to obtain BIPY-Ada $\cdot 2 \mathrm{Cl}$ dissolved in solution. Finally, BIPY-Me• $2 \mathrm{PF}_{6}$ was reprecipitated by adding solid $\mathrm{NH}_{4} \mathrm{PF}_{6}$ salt, and collected by filtration. Excess of $\mathrm{NH}_{4} \mathrm{PF}_{6}$ was removed by washing several times with $\mathrm{MeOH}$ and $\mathrm{Et}_{2} \mathrm{O}$ to obtain the pure white solid. in $\sim 38 \%$ yield. ${ }^{1} \mathrm{H}$ NMR $\left(500 \mathrm{MHz}, \mathrm{CD}_{3} \mathrm{CN}\right.$, ppm): $\delta=9.17(\mathrm{~d}, J=6.9 \mathrm{~Hz}, 4 \mathrm{H}), 8.44(\mathrm{~d}, J=6.3 \mathrm{~Hz}, 4 \mathrm{H}), 2.42(\mathrm{~m}, 6 \mathrm{H}), 2.35$ (d, $J=2.9 \mathrm{~Hz}$, 12H). 1.85 (m, 12H). ${ }^{13} \mathrm{C} \mathrm{NMR}\left(125 \mathrm{MHz}, \mathrm{CD}_{3} \mathrm{CN}, \mathrm{ppm}\right): \delta=150.2,143.4,127.9,72.3,42.7,35.5$, 31.2.

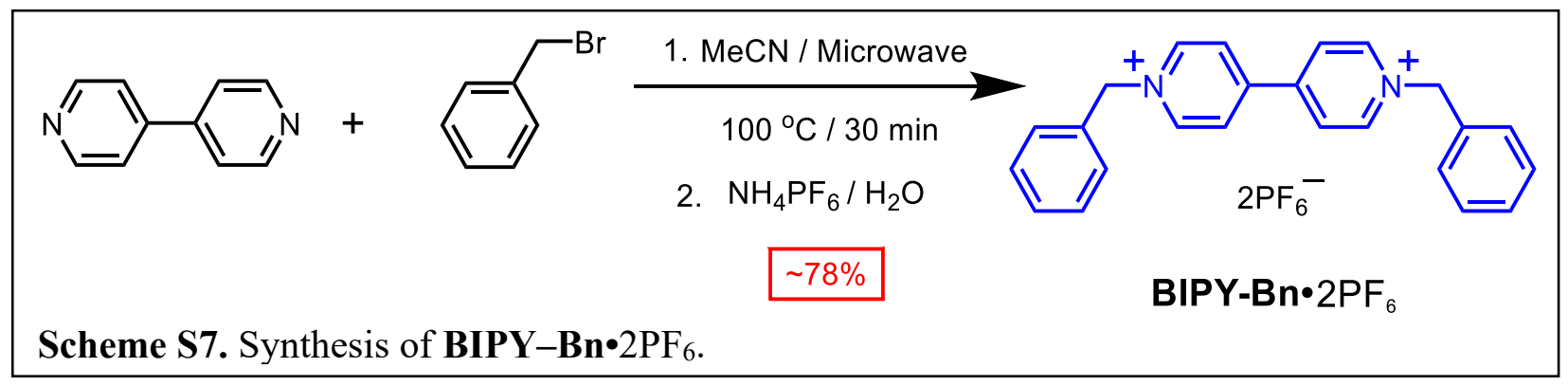

BIPY-Bn•2PF6: This compound was prepared using the same methodology as used in the preparation of BIPY-Me $2 \mathrm{PF}_{6}$. BIPY-Bn•2PF 6 was obtained as a white solid with a yield in $\sim 78 \%$. ${ }^{1} \mathrm{H}$ NMR (500 MHz, CD $\left.{ }_{3} \mathrm{CN}, \mathrm{ppm}\right): \delta=8.95(\mathrm{~d}, J=7.1 \mathrm{~Hz}, 4 \mathrm{H}), 8.35(\mathrm{~d}, J=7.1 \mathrm{~Hz}, 4 \mathrm{H}), 7.51(\mathrm{~s}$, $10 \mathrm{H}), 5.81(\mathrm{~s}, 4 \mathrm{H}) .{ }^{13} \mathrm{C} \mathrm{NMR}\left(125 \mathrm{MHz}, \mathrm{CD}_{3} \mathrm{CN}, \mathrm{ppm}\right): \delta=151.3,146.5,133.5,131.1,130.6$, $130.3,128.4,65.7$. 


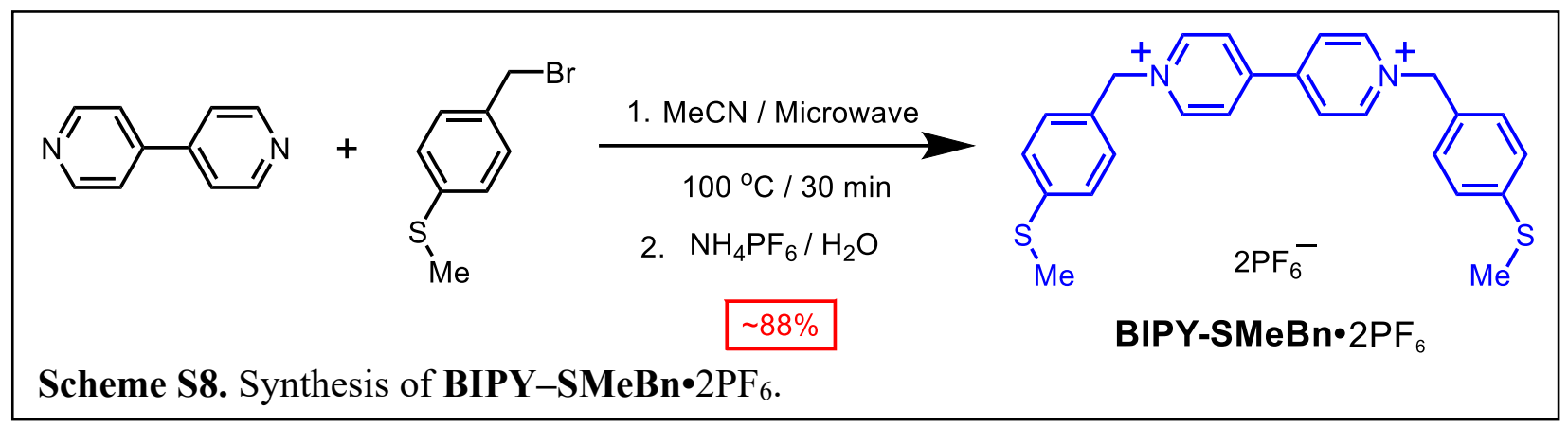

BIPY-SMeBn•2PF6: This compound was prepared using the same methodology as used in the preparation of BIPY-Me $2 \mathrm{PF}_{6}$. BIPY-SMeBn-2PF 6 was obtained as a white solid with a yield in $\sim 88 \% .{ }^{1} \mathrm{H}$ NMR $\left(500 \mathrm{MHz}, \mathrm{CD}_{3} \mathrm{CN}, \mathrm{ppm}\right): \delta=8.93(\mathrm{~d}, J=7.0 \mathrm{~Hz}, 4 \mathrm{H}), 8.33(\mathrm{~d}, J=7.0 \mathrm{~Hz}, 4 \mathrm{H})$, $7.39(\mathrm{~m}, 8 \mathrm{H}), 5.75$ (s, 4H), $2.50(\mathrm{~s}, 6 \mathrm{H}) .{ }^{13} \mathrm{C}$ NMR (125 MHz, $\left.\mathrm{CD}_{3} \mathrm{CN}, \mathrm{ppm}\right): \delta=151.2,146.4$, $142.8,130.9,129.5,128.4,127.3,65.3,15.1$.

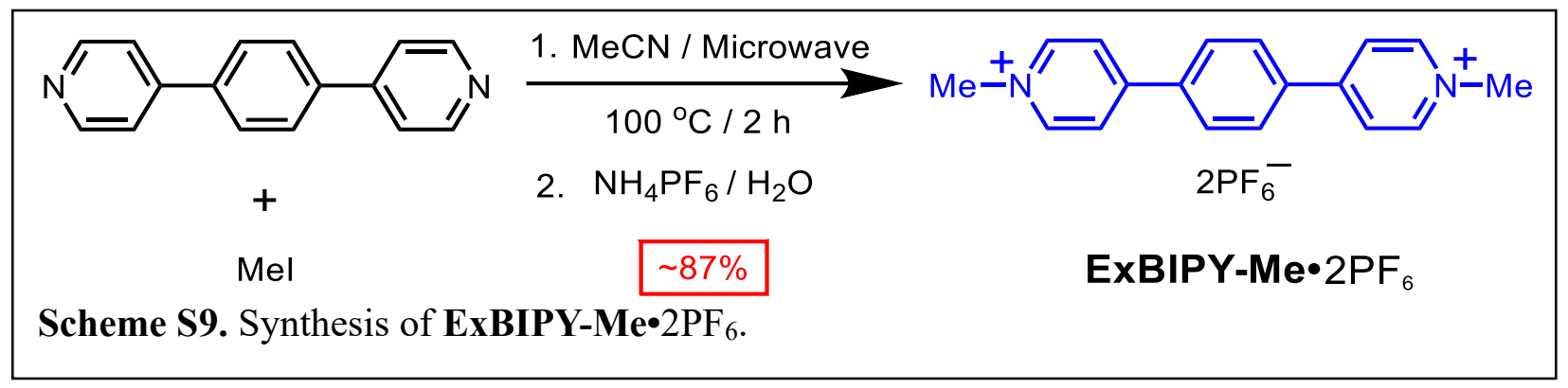

ExBIPY-Me•2PF6: This compound was prepared using the same methodology as used in the preparation of BIPY-Me $2 \mathrm{PF}_{6} \cdot \mathbf{E x B I P Y}-\mathrm{Me}^{2} 2 \mathrm{PF}_{6}$ was obtained as a white solid with a yield in $\sim 87 \% .{ }^{1} \mathrm{H}$ NMR $\left(500 \mathrm{MHz}, \mathrm{CD}_{3} \mathrm{CN}, \mathrm{ppm}\right): \delta=8.68(\mathrm{~d}, J=6.9 \mathrm{~Hz}, 4 \mathrm{H}), 8.31(\mathrm{~d}, J=6.9 \mathrm{~Hz}, 4 \mathrm{H})$, 8.13 (s, 4H), 4.32 (s, 6H). ${ }^{13} \mathrm{C}$ NMR (125 MHz, $\left.\mathrm{CD}_{3} \mathrm{CN}, \mathrm{ppm}\right): \delta=155.5,146.4,138.0,130.3$, $126.3,48.7$.

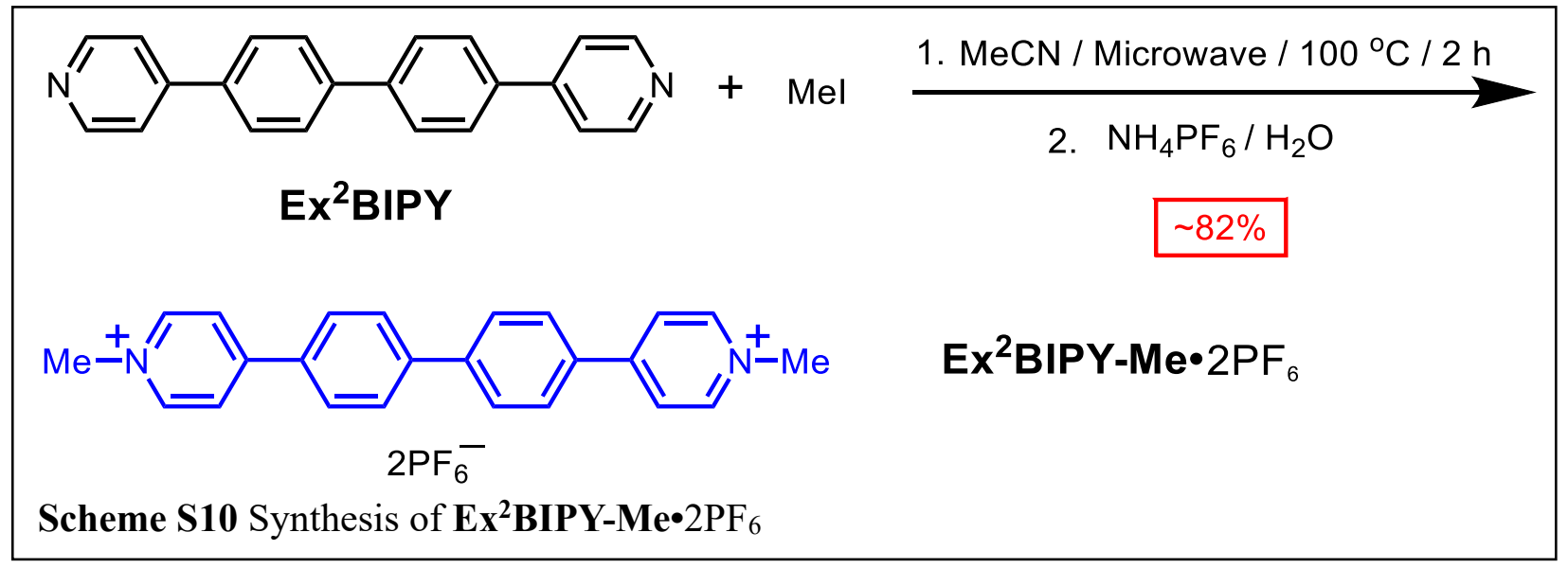


Ex² BIPY-Me•2PF6: This compound was prepared using the same methodology as used in the preparation of BIPY-Me $2 \mathrm{PF}_{6} \cdot \mathbf{E} \mathbf{x}^{2} \mathbf{B I P Y}-\mathbf{M e} \cdot 2 \mathrm{PF}_{6}$ was obtained as a white solid with a yield in $\sim 82 \% .{ }^{1} \mathrm{H}$ NMR $\left(500 \mathrm{MHz}, \mathrm{CD}_{3} \mathrm{CN}, \mathrm{ppm}\right): \delta=8.63(\mathrm{~d}, J=6.5 \mathrm{~Hz}, 4 \mathrm{H}), 8.30(\mathrm{~d}, J=6.9 \mathrm{~Hz}, 4 \mathrm{H})$, $8.05(\mathrm{~m}, 8 \mathrm{H}), 4.30(\mathrm{~s}, 6 \mathrm{H}) .{ }^{13} \mathrm{C} \mathrm{NMR}\left(125 \mathrm{MHz}, \mathrm{CD}_{3} \mathrm{CN}, \mathrm{ppm}\right): \delta=156.2,146.2,143.7,134.6$, $129.8,129.4,125.7,48.5$.

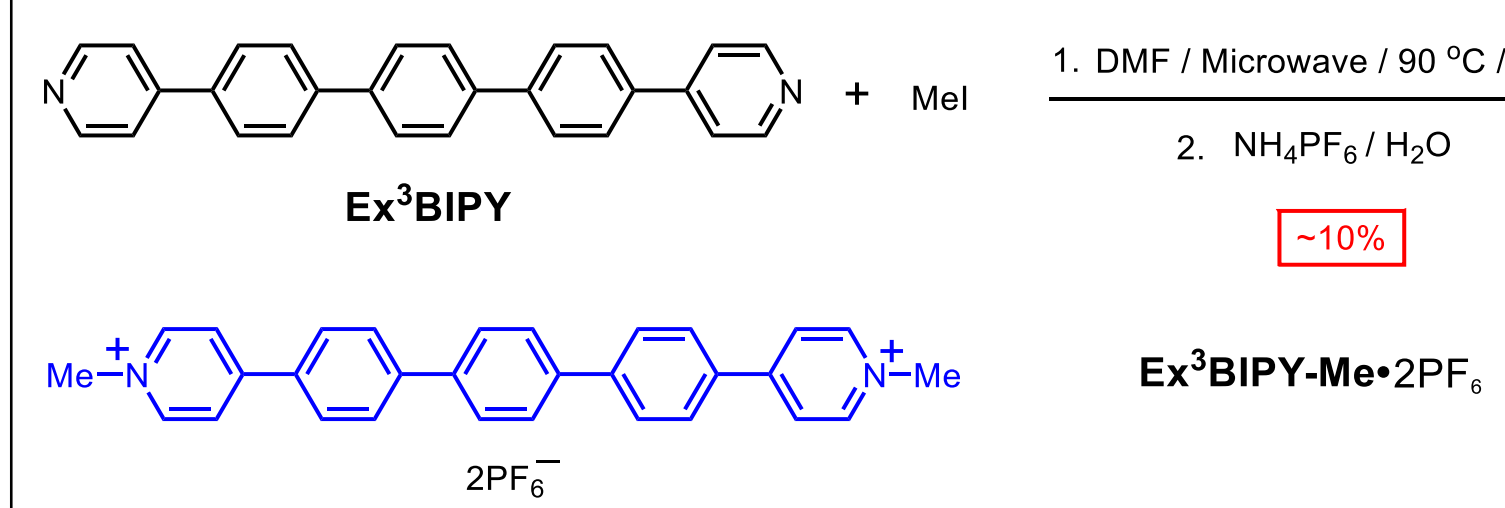

Scheme S11. Synthesis of $\mathbf{E x}^{3} \mathbf{B I P Y}-\mathrm{Me}^{2} 2 \mathrm{PF}_{6}$

BIPY-Me•2PF6: Ex³ BIPY was synthesized according to literature ${ }^{2}$. A DMF (5 mL) solution of $\mathbf{E x}^{3}$ BIPY (20 mg) and excess of MeI ( $\sim 10$ equiv) were introduced into a microwave vial. The reaction mixture was irradiated in a microwave apparatus at $90^{\circ} \mathrm{C}, 50 \mathrm{~W}$ for $2 \mathrm{~h}$. After the reaction mixture had cooled to ambient temperature, $\mathrm{H}_{2} \mathrm{O}(50 \mathrm{ml})$ was added in order to precipitate the solid BIPY-Me•2I. The BIPY-Me•2I solid was filtered off, washed with $\mathrm{Et}_{2} \mathrm{O}$, and dissolved in $\mathrm{MeOH}$ $(\sim 30 \mathrm{~mL})$. Finally, the BIPY-Me $\cdot 2 \mathrm{PF}_{6}$ was reprecipitated by adding solid $\mathrm{NH}_{4} \mathrm{PF}_{6}$ salt, and collected by filtration. Excess of $\mathrm{NH}_{4} \mathrm{PF}_{6}$ was removed by washing several times with $\mathrm{MeOH}$ and $\mathrm{Et}_{2} \mathrm{O}$ to obtain the pure white solid. in $\sim 10 \%$ yield. ${ }^{1} \mathrm{H}$ NMR $\left(500 \mathrm{MHz}, \mathrm{CD}_{3} \mathrm{CN}, \mathrm{ppm}\right): \delta=8.64$ $(\mathrm{d}, J=6.7 \mathrm{~Hz}, 4 \mathrm{H}), 8.33(\mathrm{~d}, J=6.5 \mathrm{~Hz}, 4 \mathrm{H}),), 8.06(\mathrm{~m}, 8 \mathrm{H}), 7.96(\mathrm{~s}, 6 \mathrm{H}), 4.32(\mathrm{~s}, 6 \mathrm{H}) .{ }^{13} \mathrm{C} \mathrm{NMR}$ (125 MHz, $\left.\mathrm{CD}_{3} \mathrm{CN}, \mathrm{ppm}\right): \delta=156.3,146.1,144.7,140.1,133.8,129.7,129.1,128.8,125.5,48.4$. 


\section{Section C. NMR Spectroscopy}

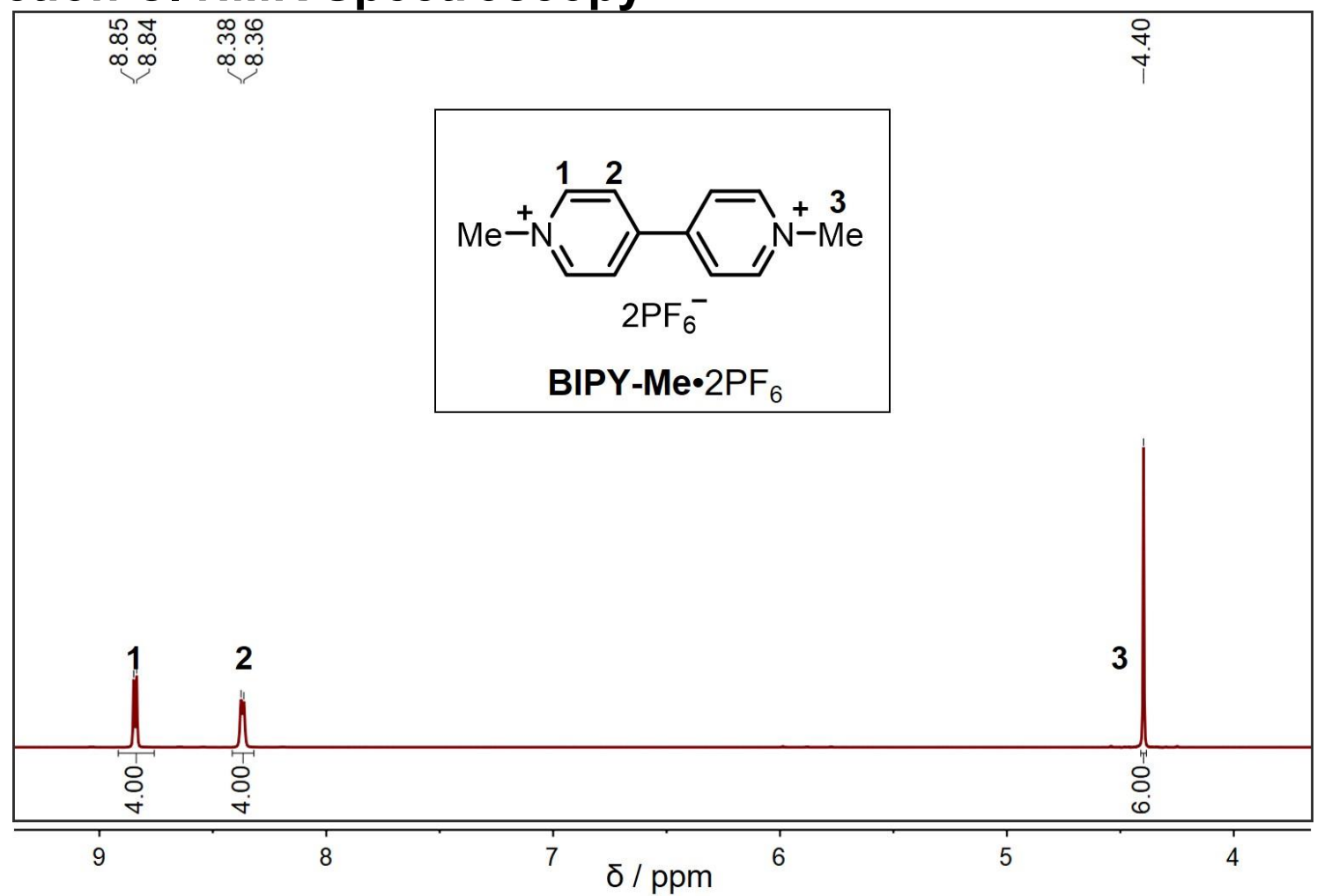

Figure S1. ${ }^{1} \mathrm{H}$ NMR Spectrum $\left(500 \mathrm{MHz}, \mathrm{CD}_{3} \mathrm{CN}, 298 \mathrm{~K}\right)$ of BIPY-Me•2PF 6

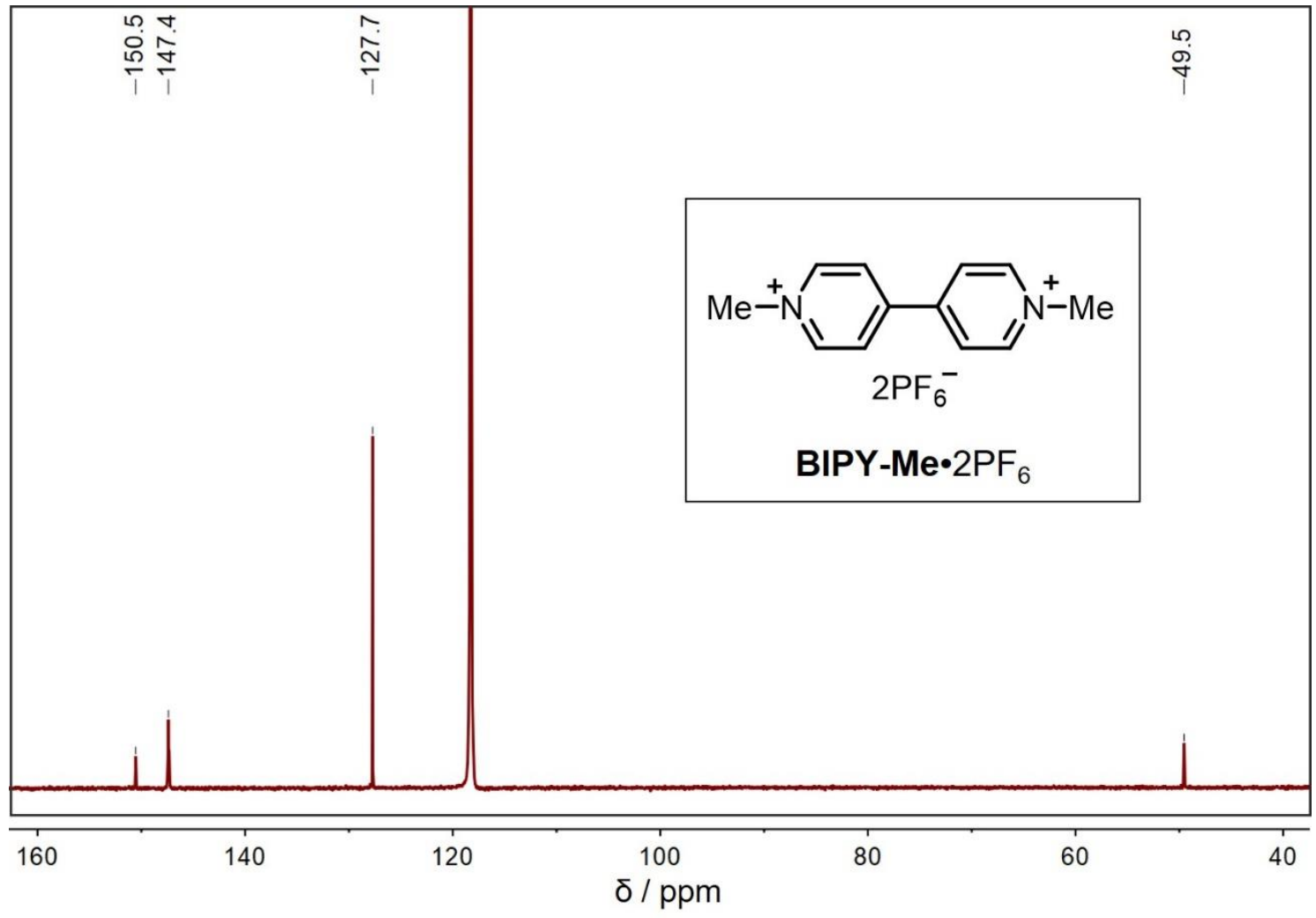

Figure S2. ${ }^{13} \mathrm{C}$ NMR Spectrum $\left(125 \mathrm{MHz}, \mathrm{CD}_{3} \mathrm{CN}, 298 \mathrm{~K}\right)$ of BIPY-Me $2 \mathrm{PF}_{6}$ 


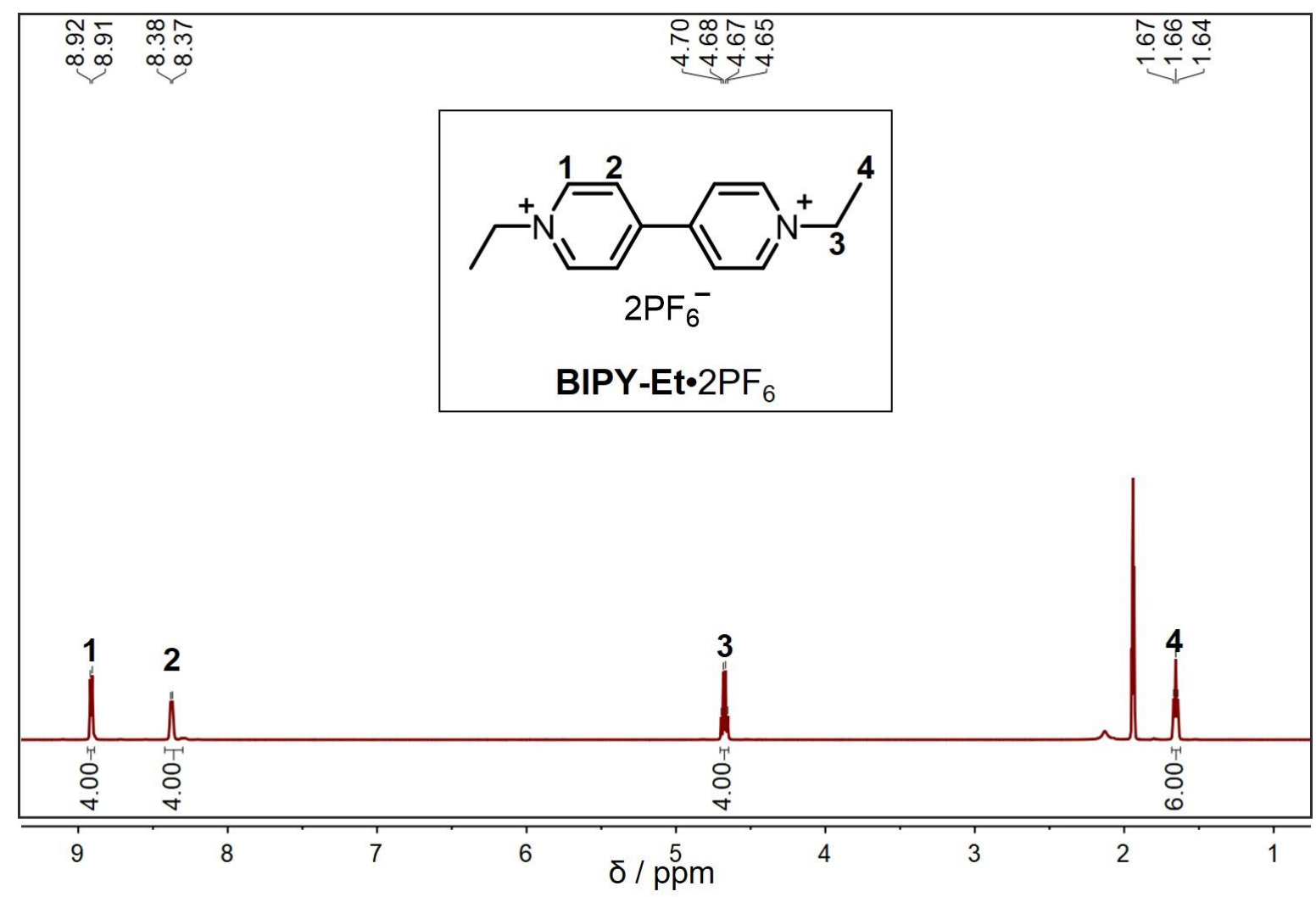

Figure S3. ${ }^{1} \mathrm{H}$ NMR Spectrum $\left(500 \mathrm{MHz}, \mathrm{CD}_{3} \mathrm{CN}, 298 \mathrm{~K}\right)$ of BIPY-Et• $2 \mathrm{PF}_{6}$

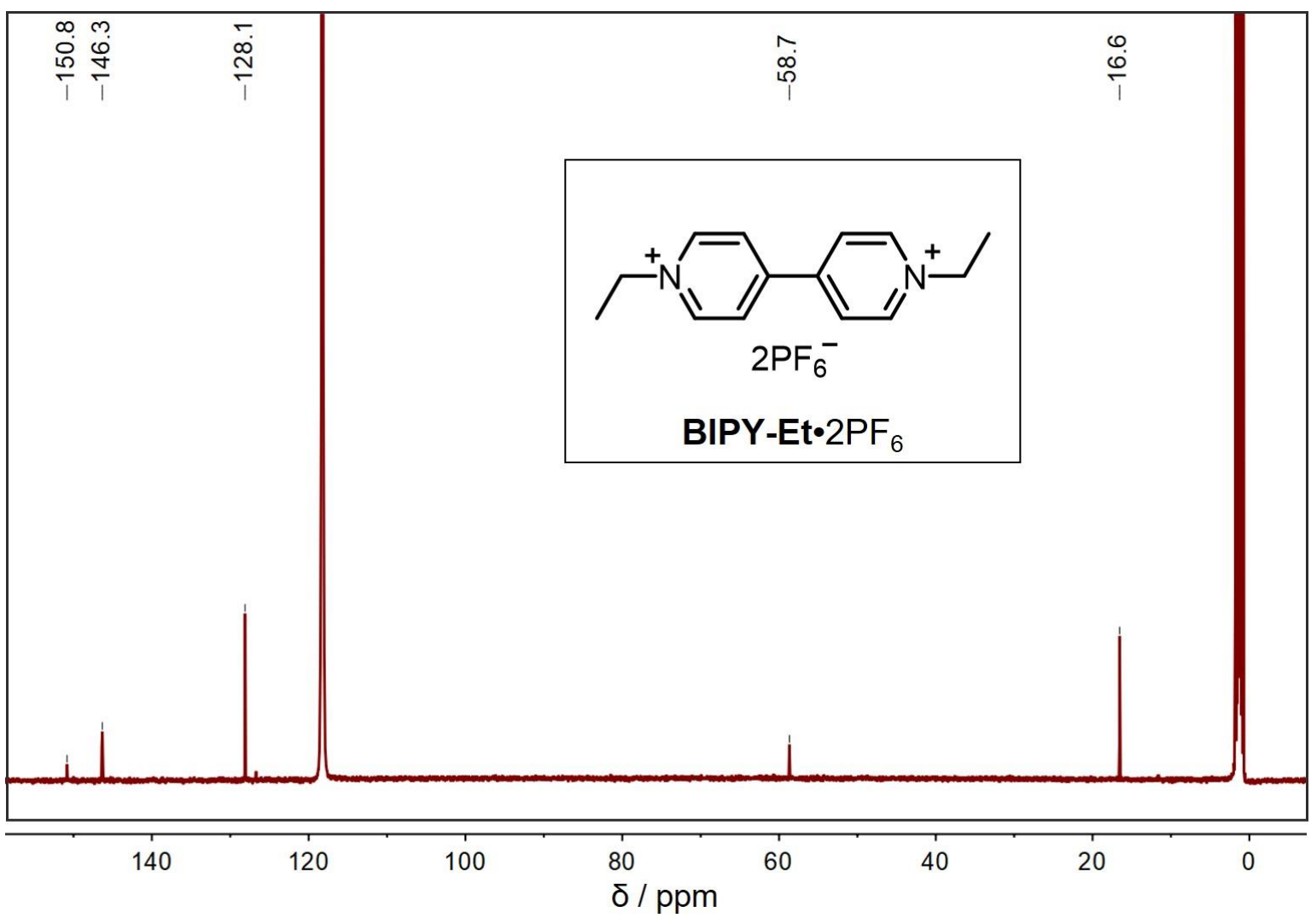

Figure S4. ${ }^{13} \mathrm{C}$ NMR Spectrum $\left(125 \mathrm{MHz}, \mathrm{CD}_{3} \mathrm{CN}, 298 \mathrm{~K}\right)$ of BIPY-Et•2PF 6 


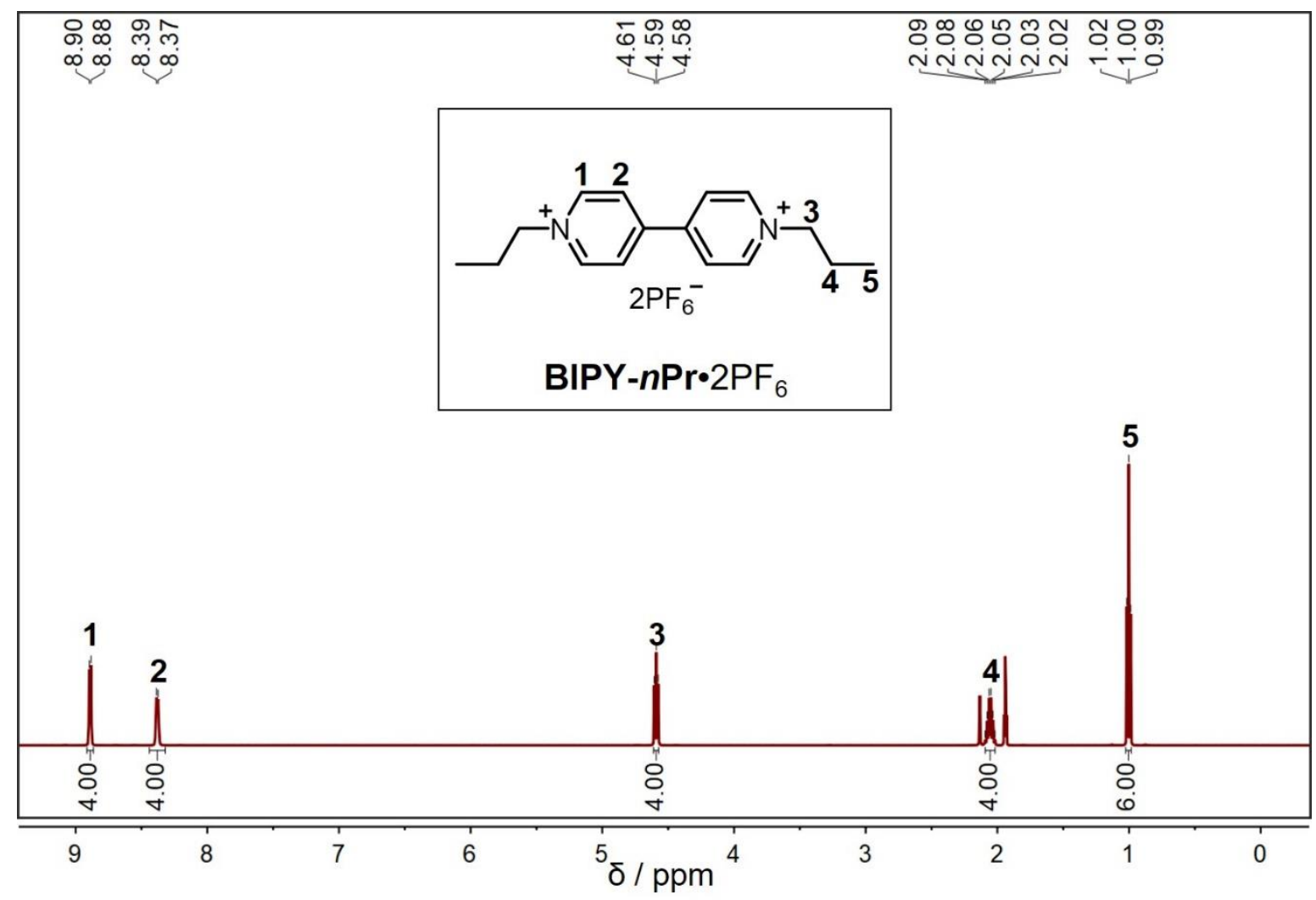

Figure S5. ${ }^{1} \mathrm{H}$ NMR Spectrum (500 MHz, CD $\left.{ }_{3} \mathrm{CN}, 298 \mathrm{~K}\right)$ of BIPY-nPr•2PF 6

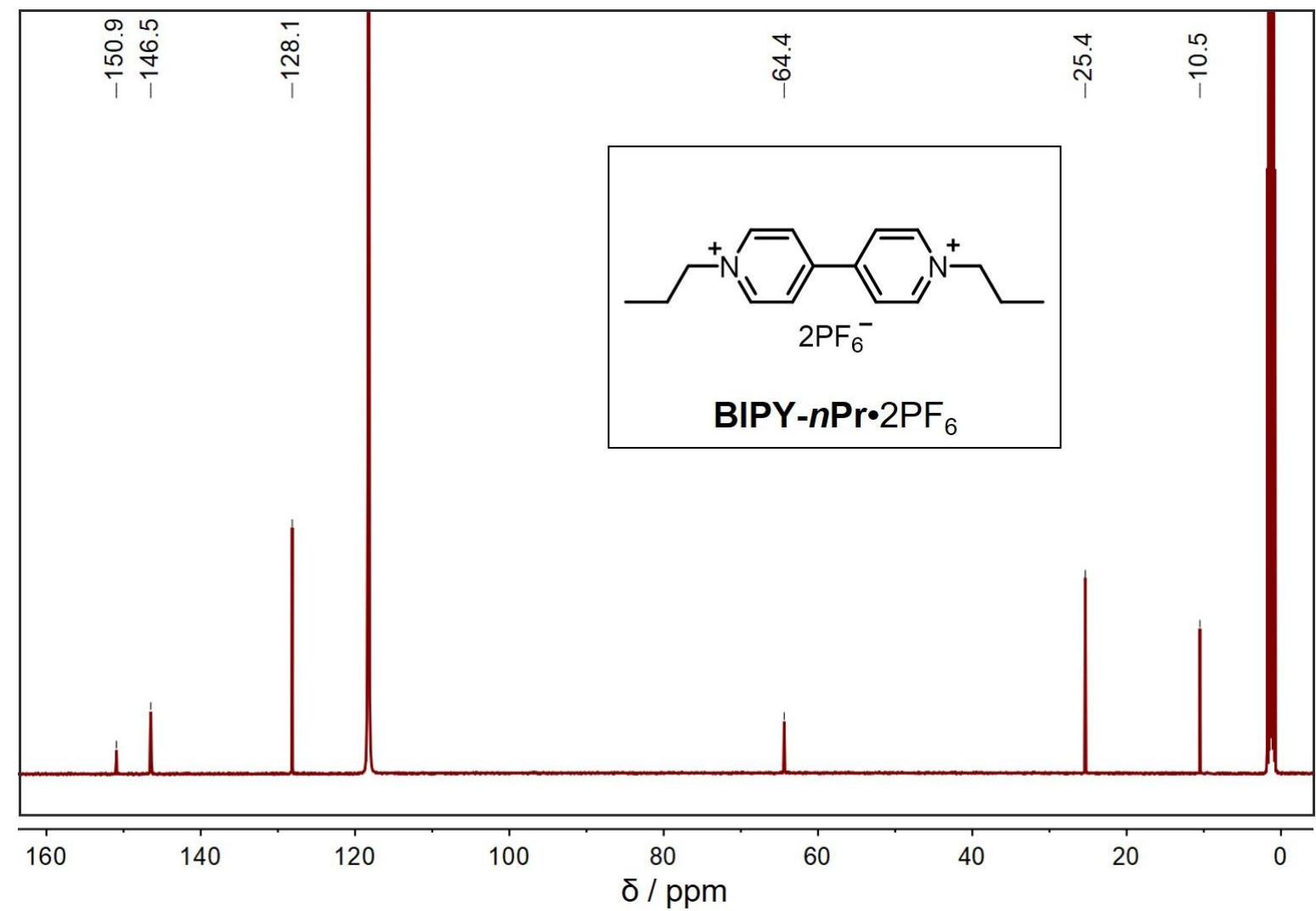

Figure S6. ${ }^{13} \mathrm{C}$ NMR Spectrum $\left(125 \mathrm{MHz}, \mathrm{CD}_{3} \mathrm{CN}, 298 \mathrm{~K}\right)$ of BIPY-nPr$\cdot 2 \mathrm{PF}_{6}$ 


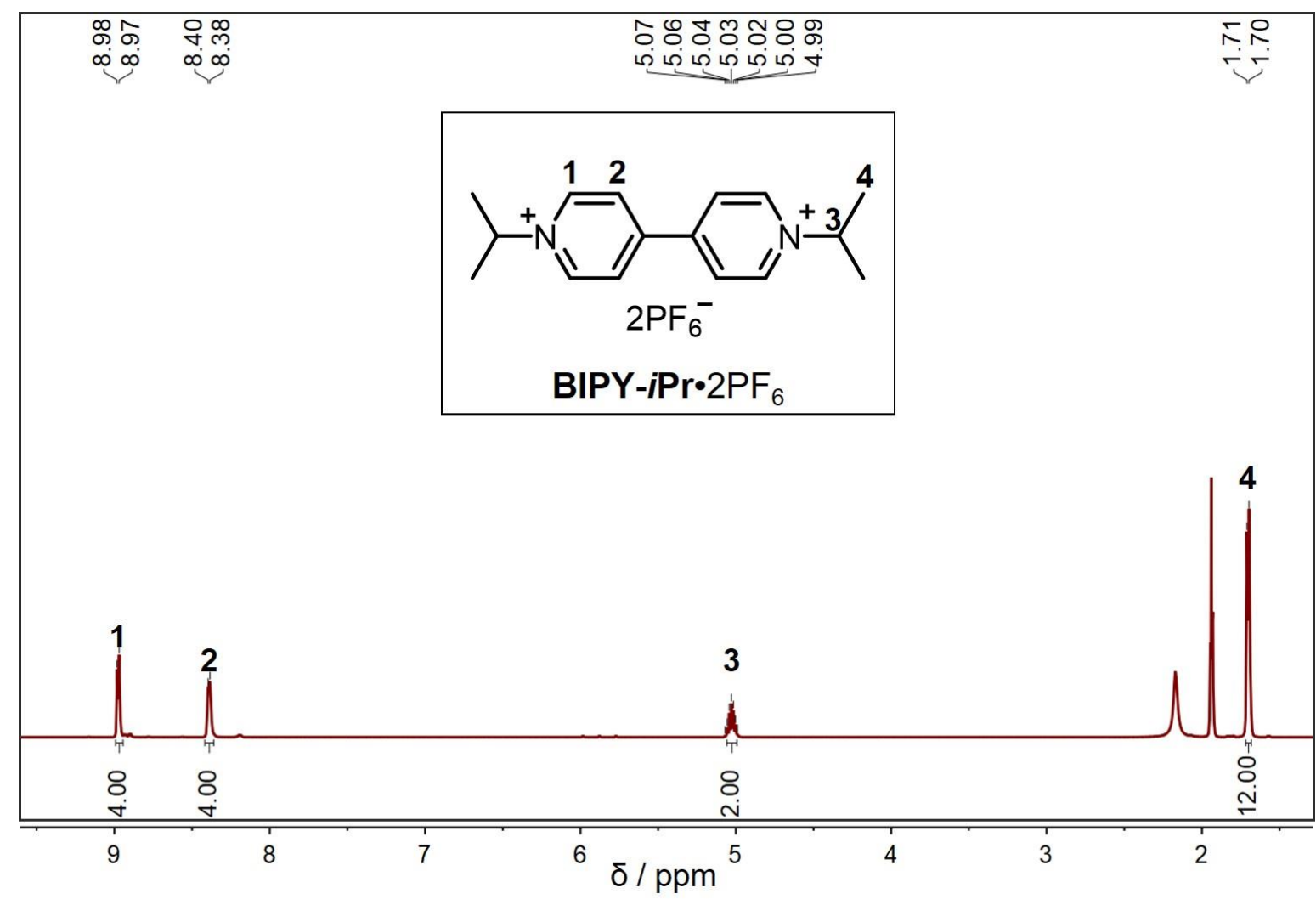

Figure S7. ${ }^{1} \mathrm{H}$ NMR Spectrum $\left(500 \mathrm{MHz}, \mathrm{CD}_{3} \mathrm{CN}, 298 \mathrm{~K}\right)$ of BIPY-iPr•2PF 6

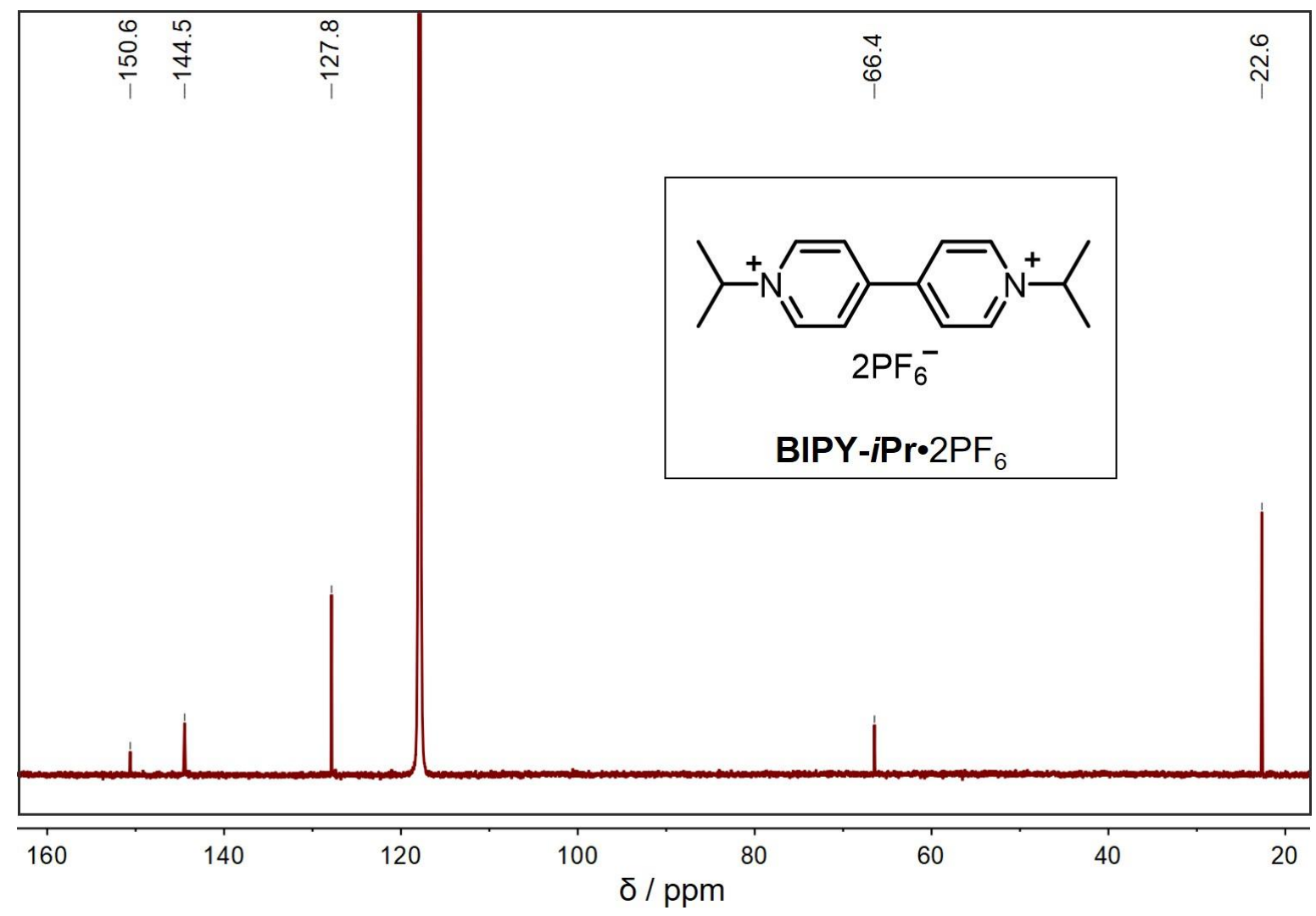

Figure S8. ${ }^{13} \mathrm{C}$ NMR Spectrum $\left(125 \mathrm{MHz}, \mathrm{CD}_{3} \mathrm{CN}, 298 \mathrm{~K}\right)$ of BIPY-iPr$\cdot 2 \mathrm{PF}_{6}$ 


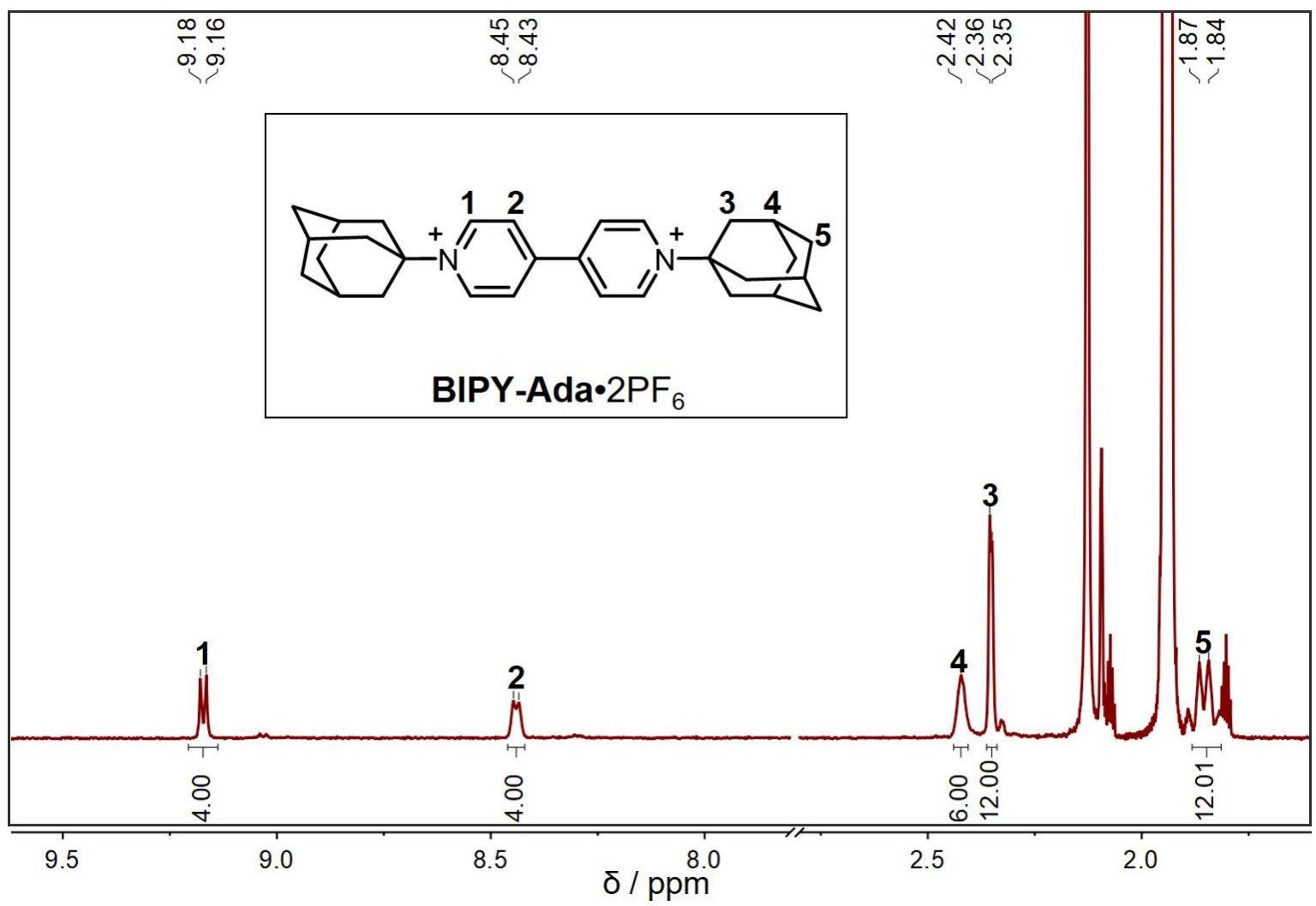

Figure S9. ${ }^{1} \mathrm{H}$ NMR Spectrum $\left(500 \mathrm{MHz}, \mathrm{CD}_{3} \mathrm{CN}, 298 \mathrm{~K}\right)$ of BIPY-Ada $2 \mathrm{PF}_{6}$

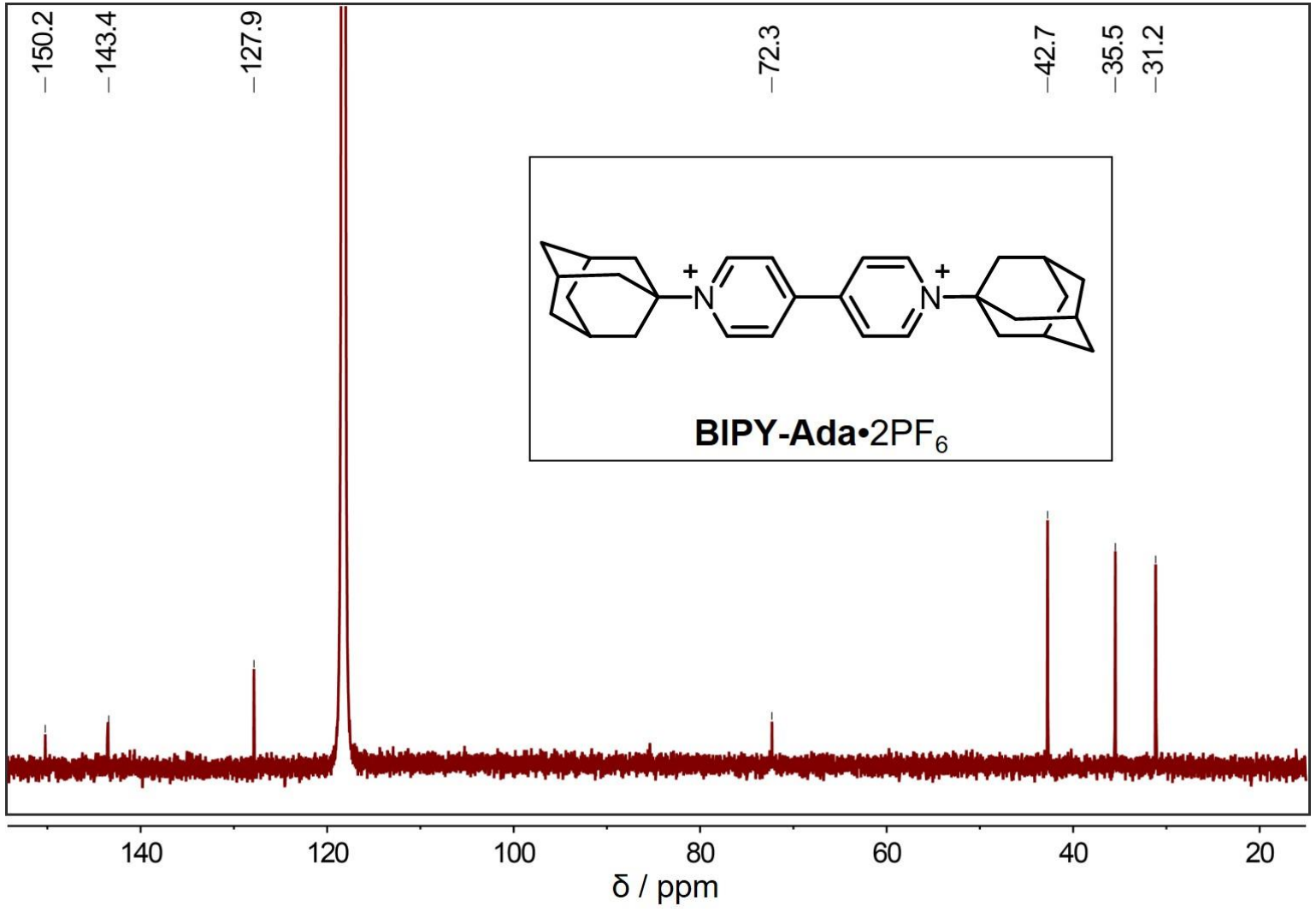

Figure S10. ${ }^{13} \mathrm{C}$ NMR Spectrum $\left(125 \mathrm{MHz}, \mathrm{CD}_{3} \mathrm{CN}, 298 \mathrm{~K}\right)$ of BIPY-Ada-2PF 6 


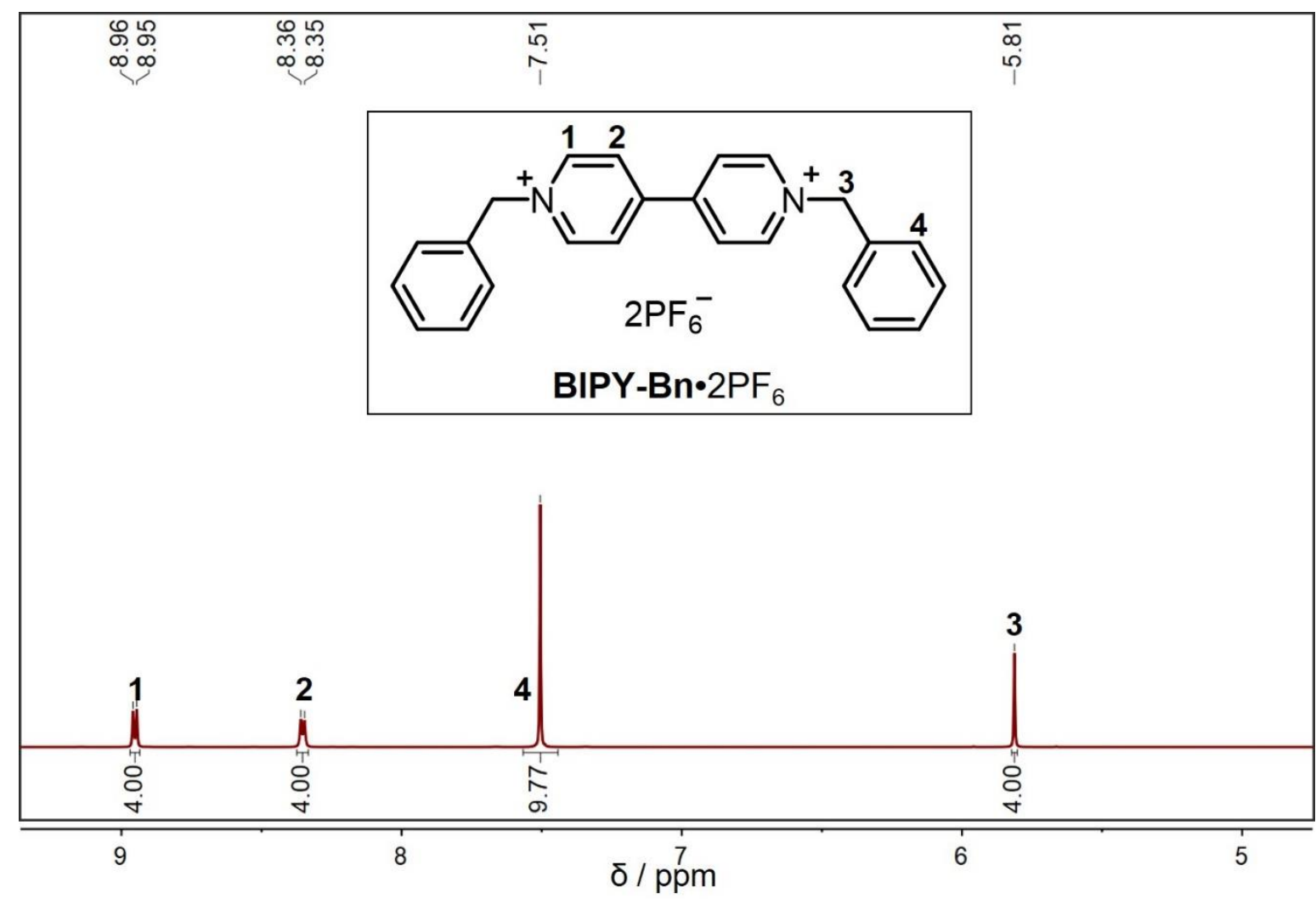

Figure S11. ${ }^{1} \mathrm{H}$ NMR Spectrum $\left(500 \mathrm{MHz}, \mathrm{CD}_{3} \mathrm{CN}, 298 \mathrm{~K}\right)$ of BIPY-Bn・2PF 6

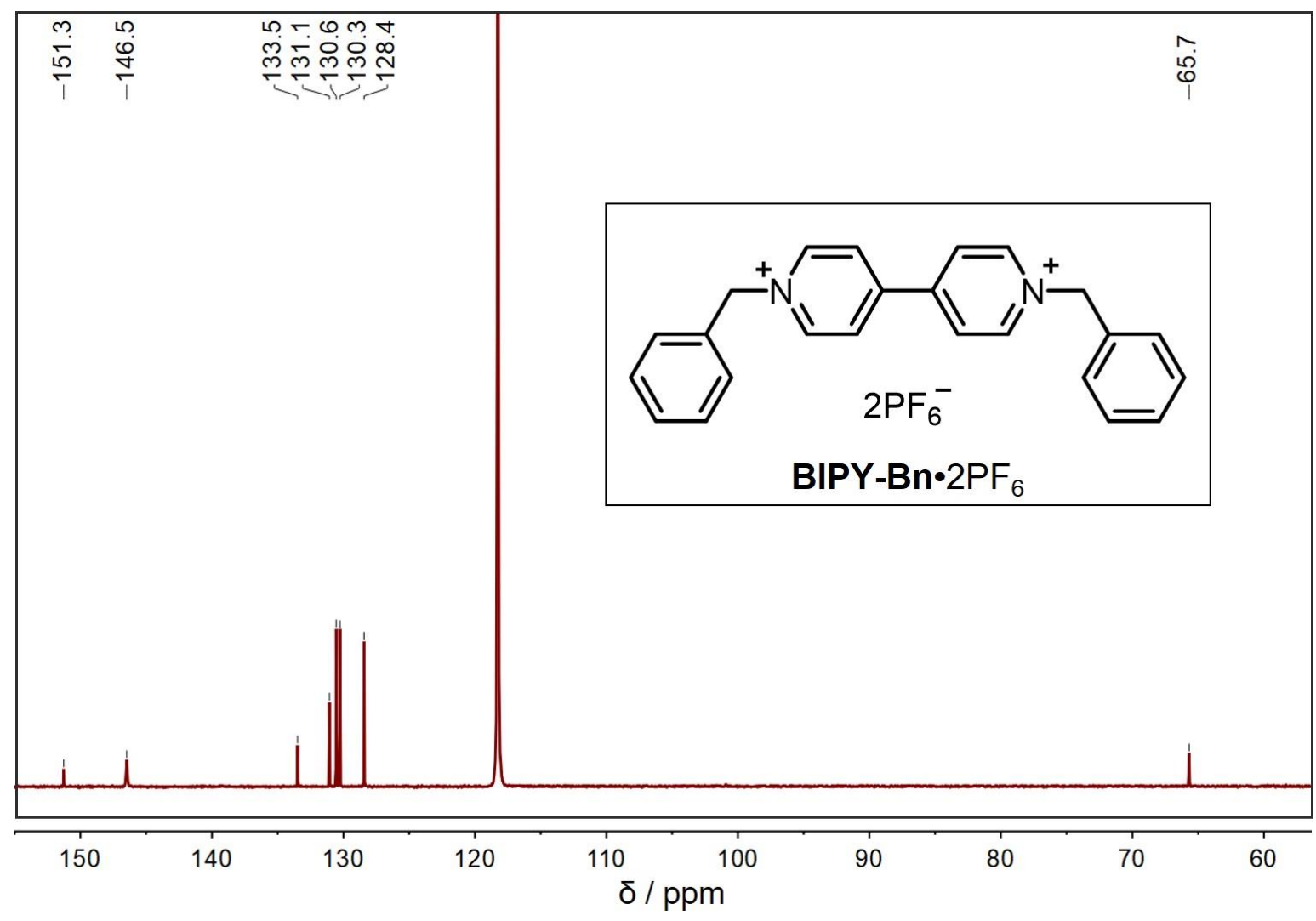

Figure S12. ${ }^{13} \mathrm{C}$ NMR Spectrum $\left(125 \mathrm{MHz}, \mathrm{CD}_{3} \mathrm{CN}, 298 \mathrm{~K}\right)$ of BIPY-Bn•2PF 6 


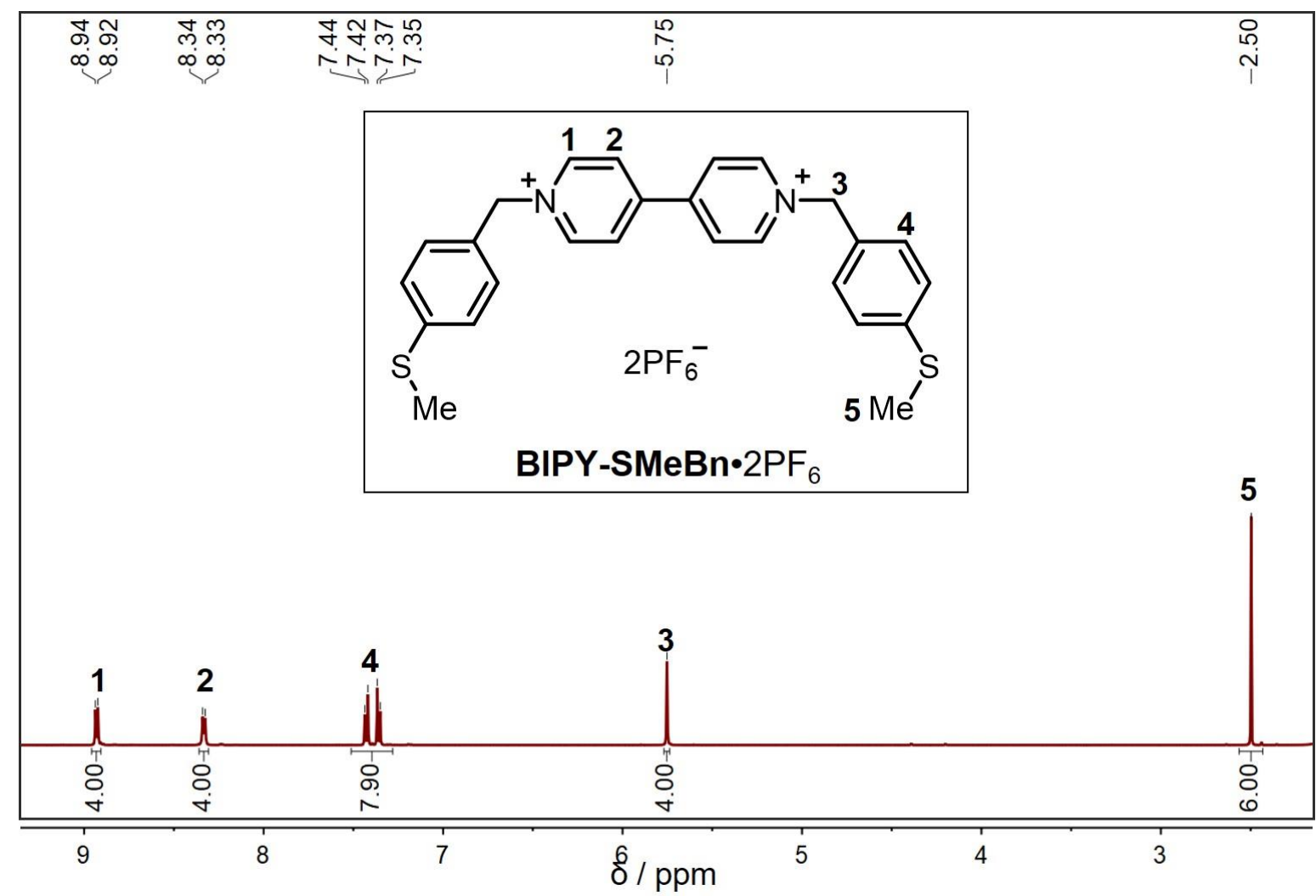

Figure S13. ${ }^{1} \mathrm{H}$ NMR Spectrum (500 MHz, CD ${ }_{3} \mathrm{CN}, 298 \mathrm{~K}$ ) of BIPY-SMeBn・2PF 6

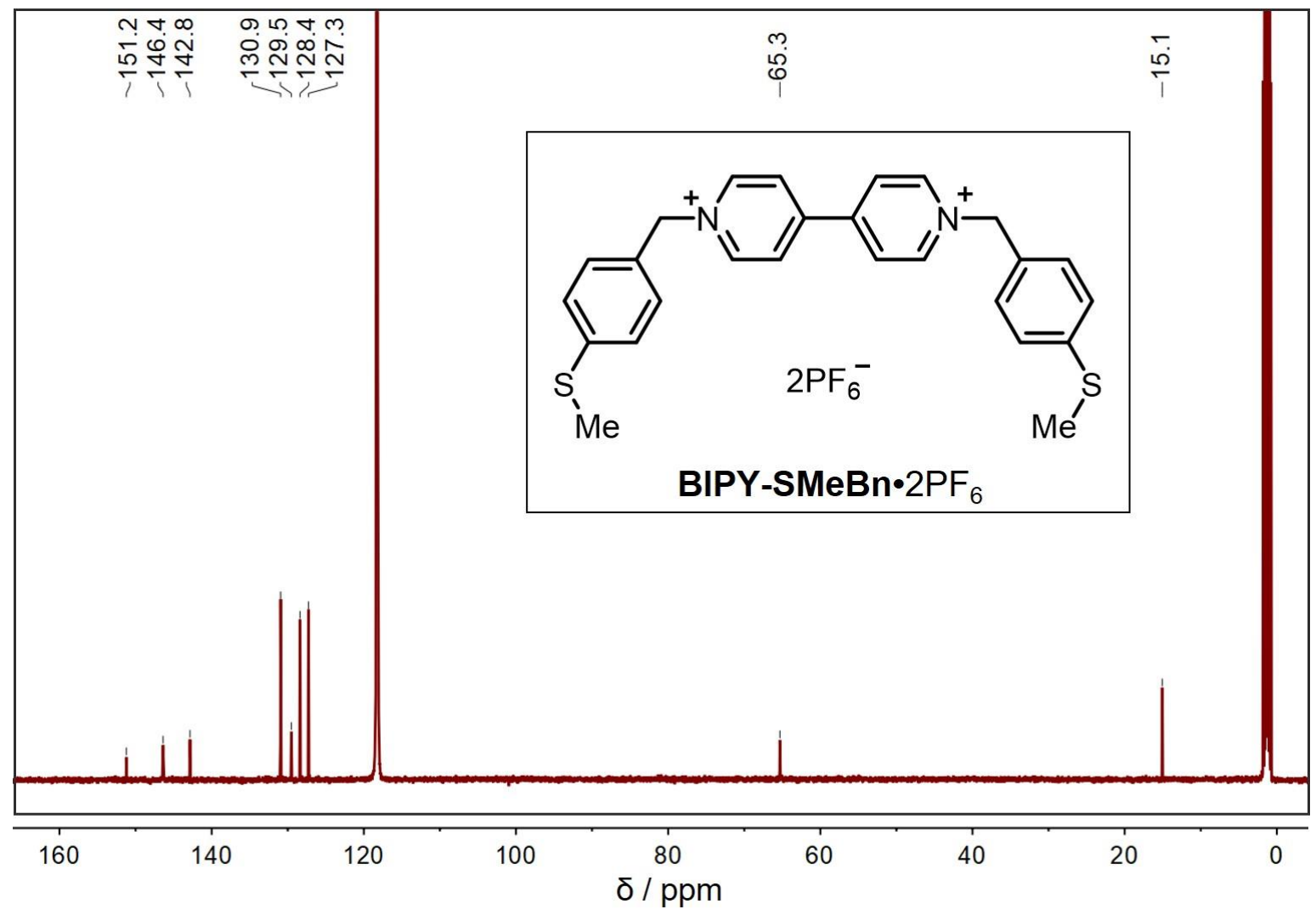

Figure S14. ${ }^{13} \mathrm{C}$ NMR Spectrum $\left(125 \mathrm{MHz}, \mathrm{CD}_{3} \mathrm{CN}, 298 \mathrm{~K}\right)$ of BIPY-SMeBn•2PF 6 


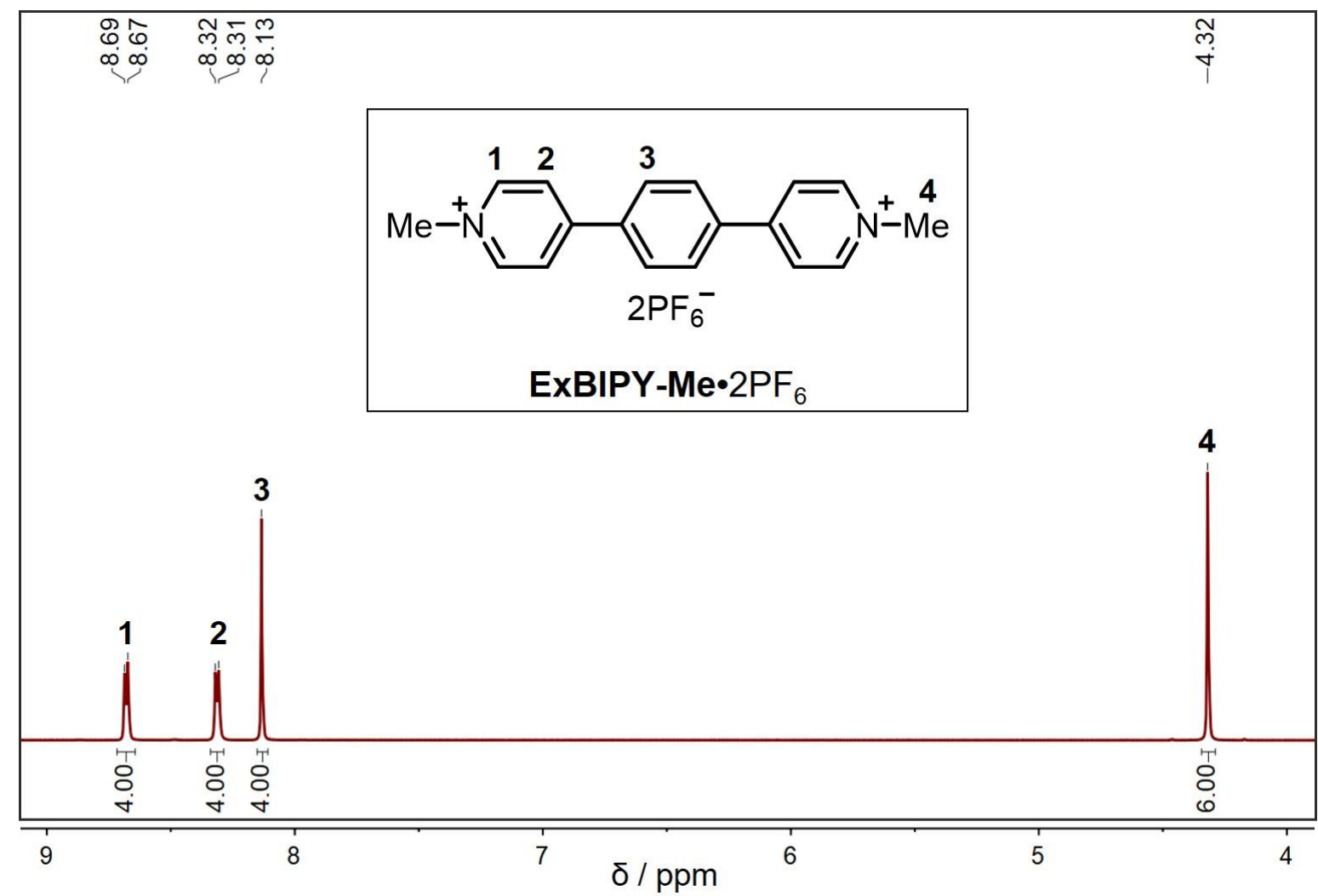

Figure S15. ${ }^{1} \mathrm{H}$ NMR Spectrum $\left(500 \mathrm{MHz}, \mathrm{CD}_{3} \mathrm{CN}, 298 \mathrm{~K}\right)$ of ExBIPY-Me•2PF 6

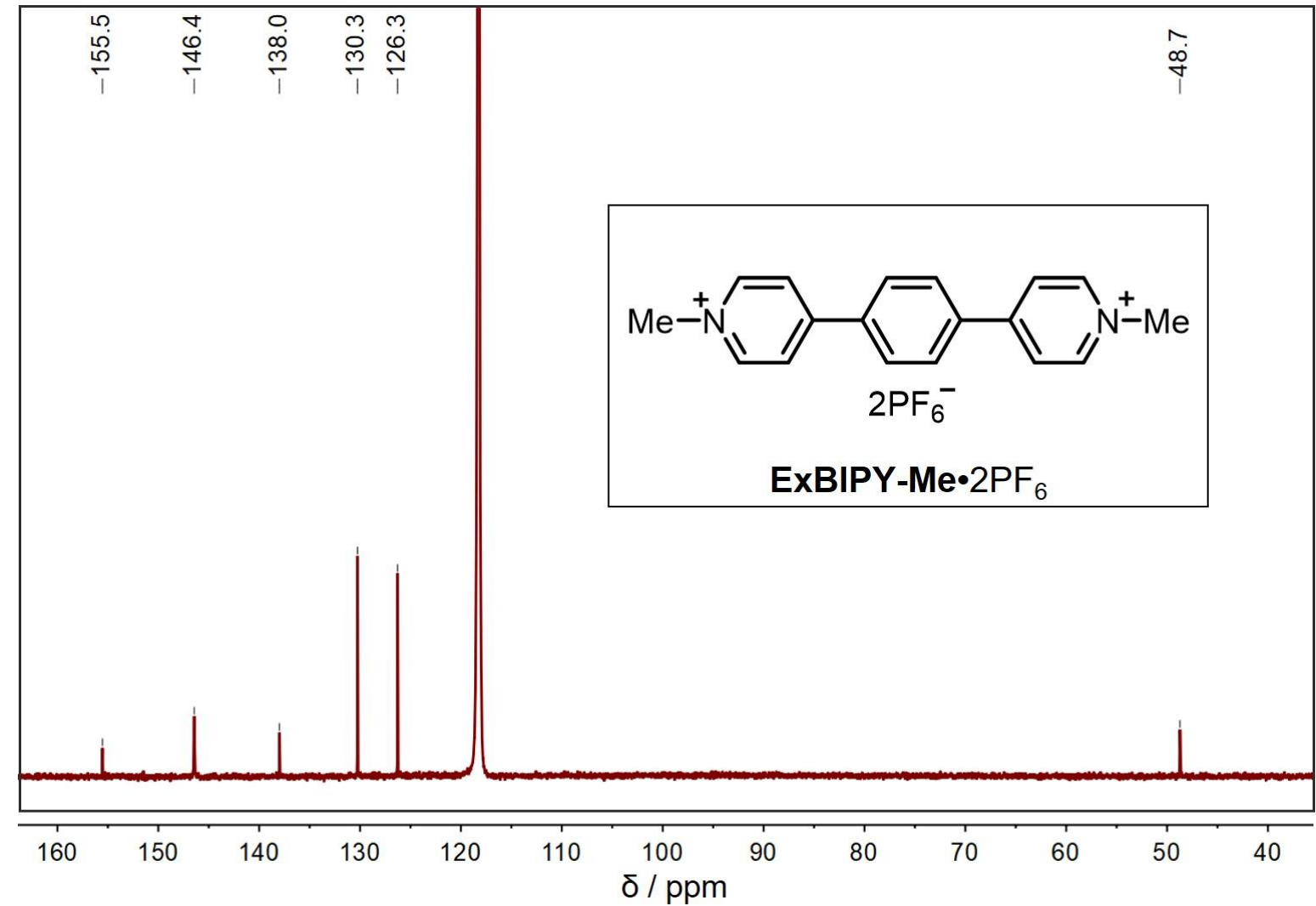

Figure S16. ${ }^{13} \mathrm{C}$ NMR Spectrum $\left(125 \mathrm{MHz}, \mathrm{CD}_{3} \mathrm{CN}, 298 \mathrm{~K}\right)$ of ExBIPY-Me•2PF 6 S16 


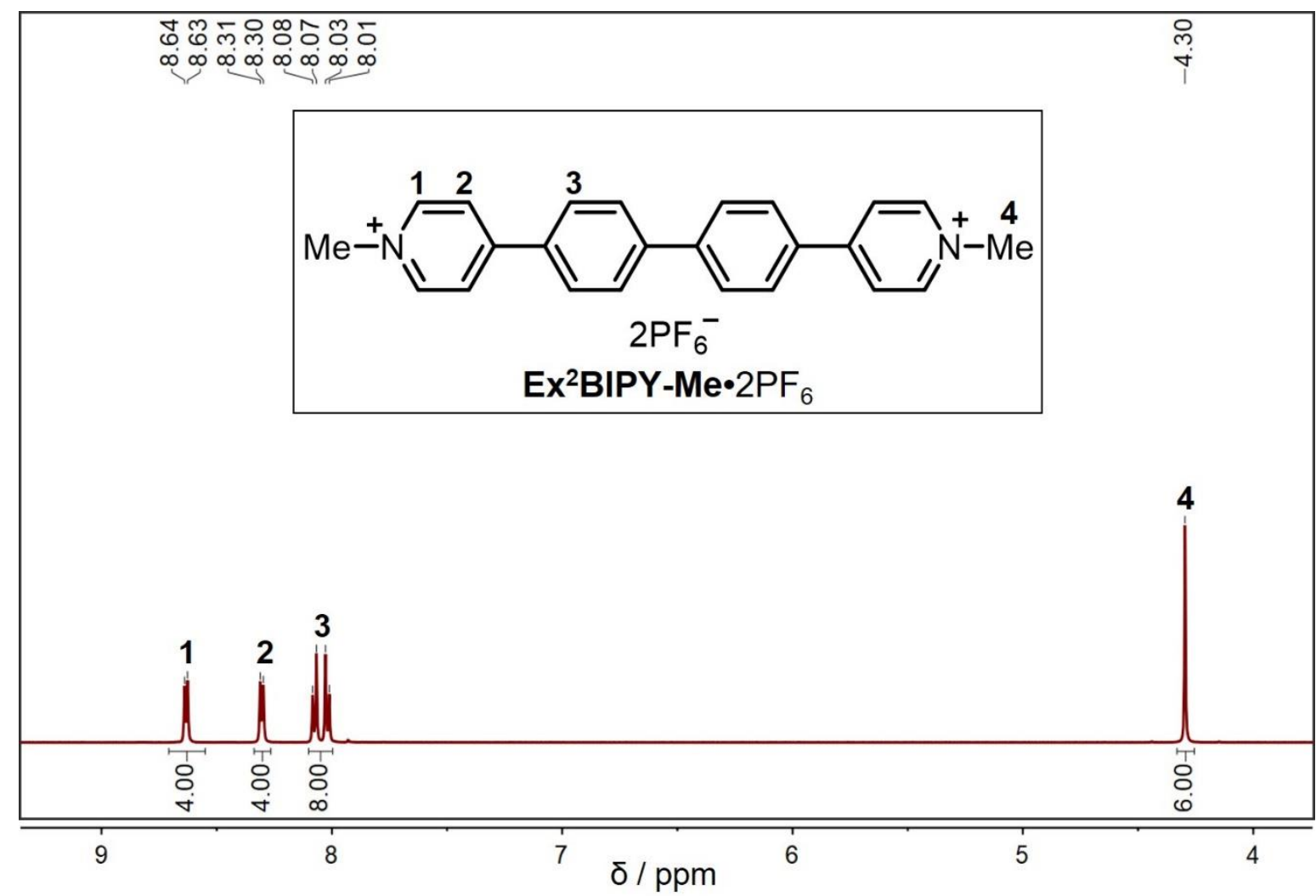

Figure S17. ${ }^{1} \mathrm{H}$ NMR Spectrum (500 MHz, CD 3 CN, $298 \mathrm{~K}$ ) of $\mathbf{E x}^{2} \mathbf{B I P Y}-\mathbf{M e} \cdot 2 \mathrm{PF}_{6}$

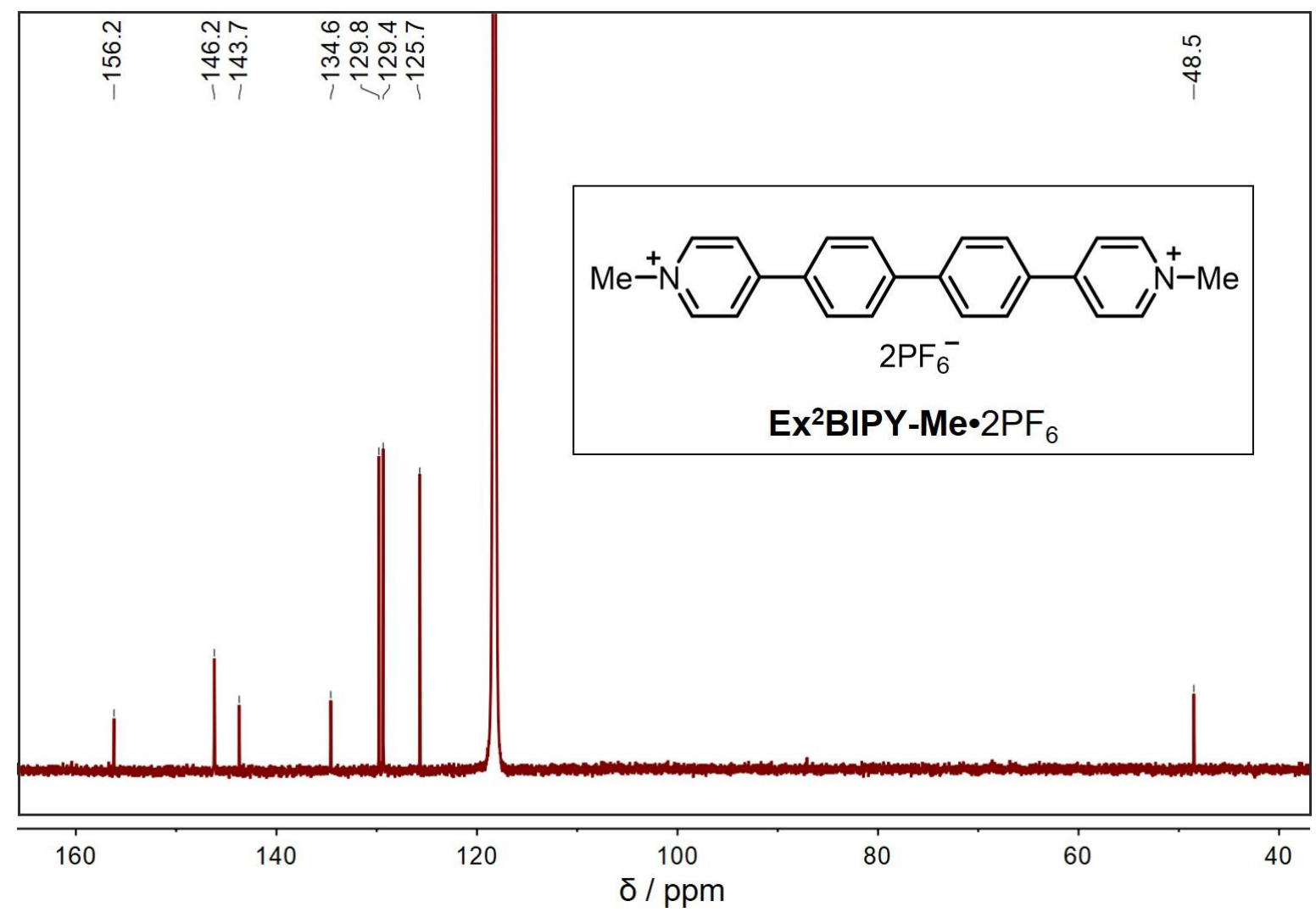

Figure S18. ${ }^{13} \mathrm{C}$ NMR Spectrum $\left(125 \mathrm{MHz}, \mathrm{CD}_{3} \mathrm{CN}, 298 \mathrm{~K}\right)$ of $\mathbf{E x} \mathbf{x}^{2} \mathbf{B I P Y}-\mathbf{M e} \cdot 2 \mathrm{PF}_{6}$ 


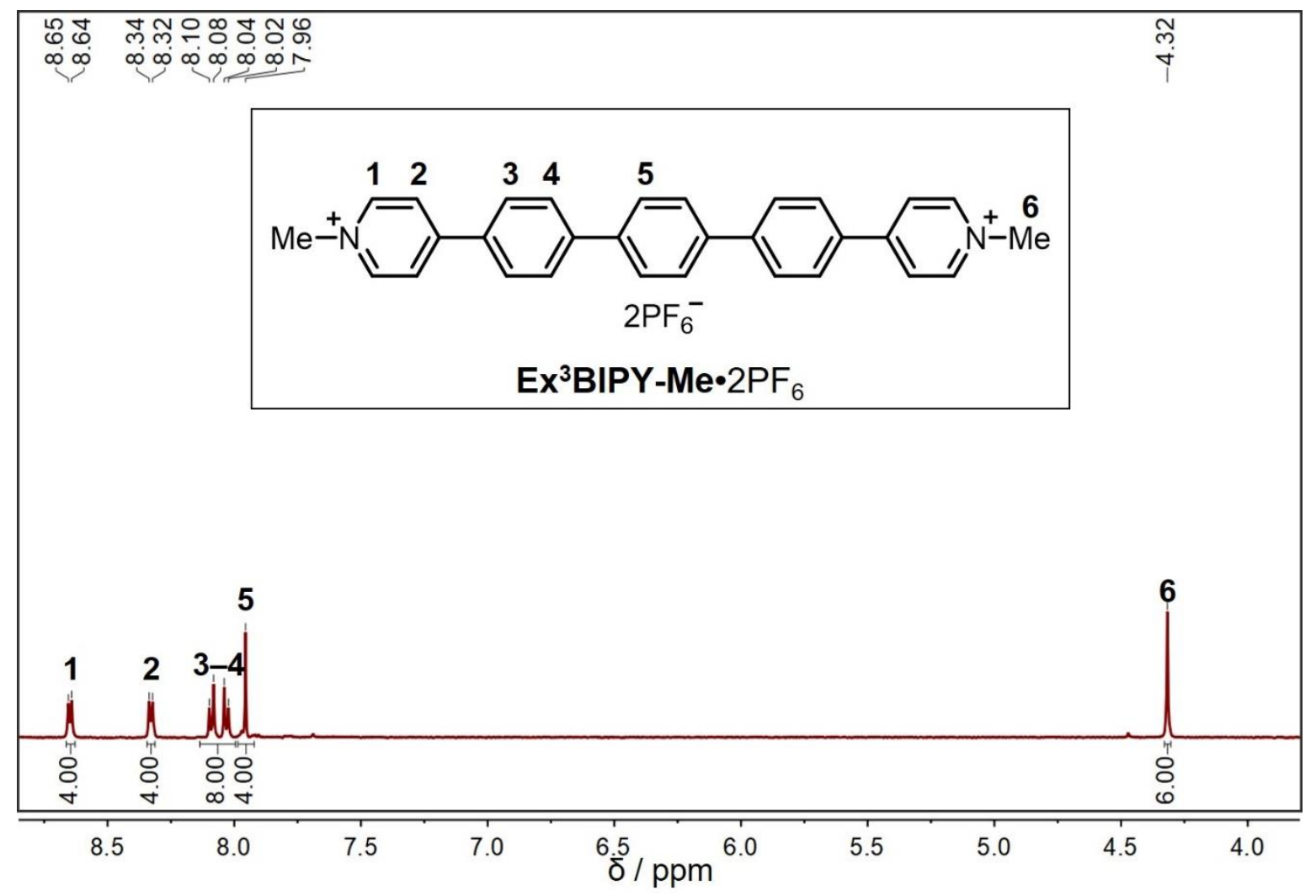

Figure S19. ${ }^{1} \mathrm{H}$ NMR Spectrum (500 MHz, CD $\left.{ }_{3} \mathrm{CN}, 298 \mathrm{~K}\right)$ of $\mathbf{E x}^{\mathbf{3} B I P Y}-\mathbf{M e} \cdot 2 \mathrm{PF}_{6}$

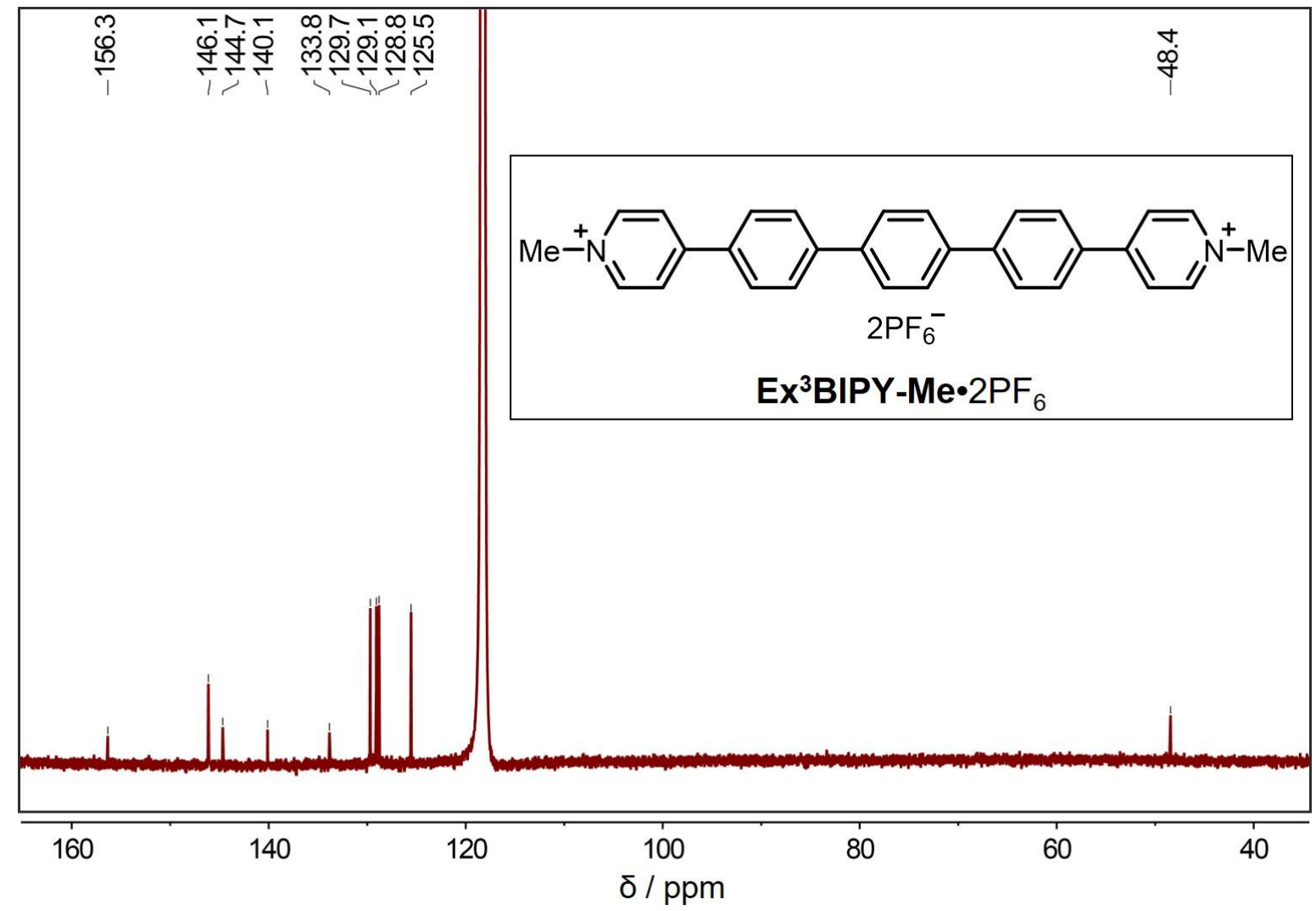

Figure S20. ${ }^{13} \mathrm{C}$ NMR Spectrum $\left(125 \mathrm{MHz}, \mathrm{CD}_{3} \mathrm{CN}, 298 \mathrm{~K}\right)$ of $\mathbf{E x}^{\mathbf{3}} \mathbf{B I P Y}-\mathbf{M e} \cdot 2 \mathrm{PF}_{6}$ 


\section{Section D. Supplemental Characterizations}
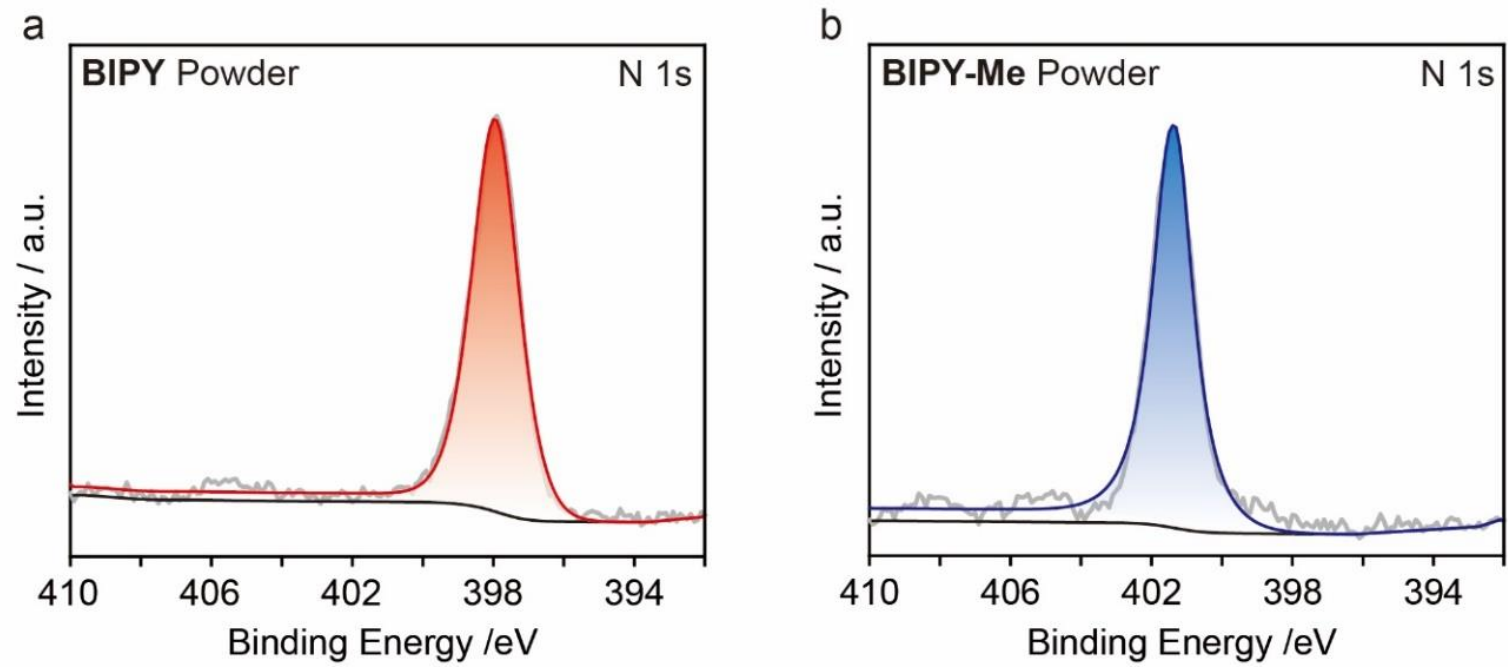

Figure S21. X-ray photoelectron spectroscopy (XPS) characterizations showing the N(1s) region of the BIPY (a) and BIPY-Me $2 \mathrm{PF}_{6}(\mathrm{~b})$ powders.
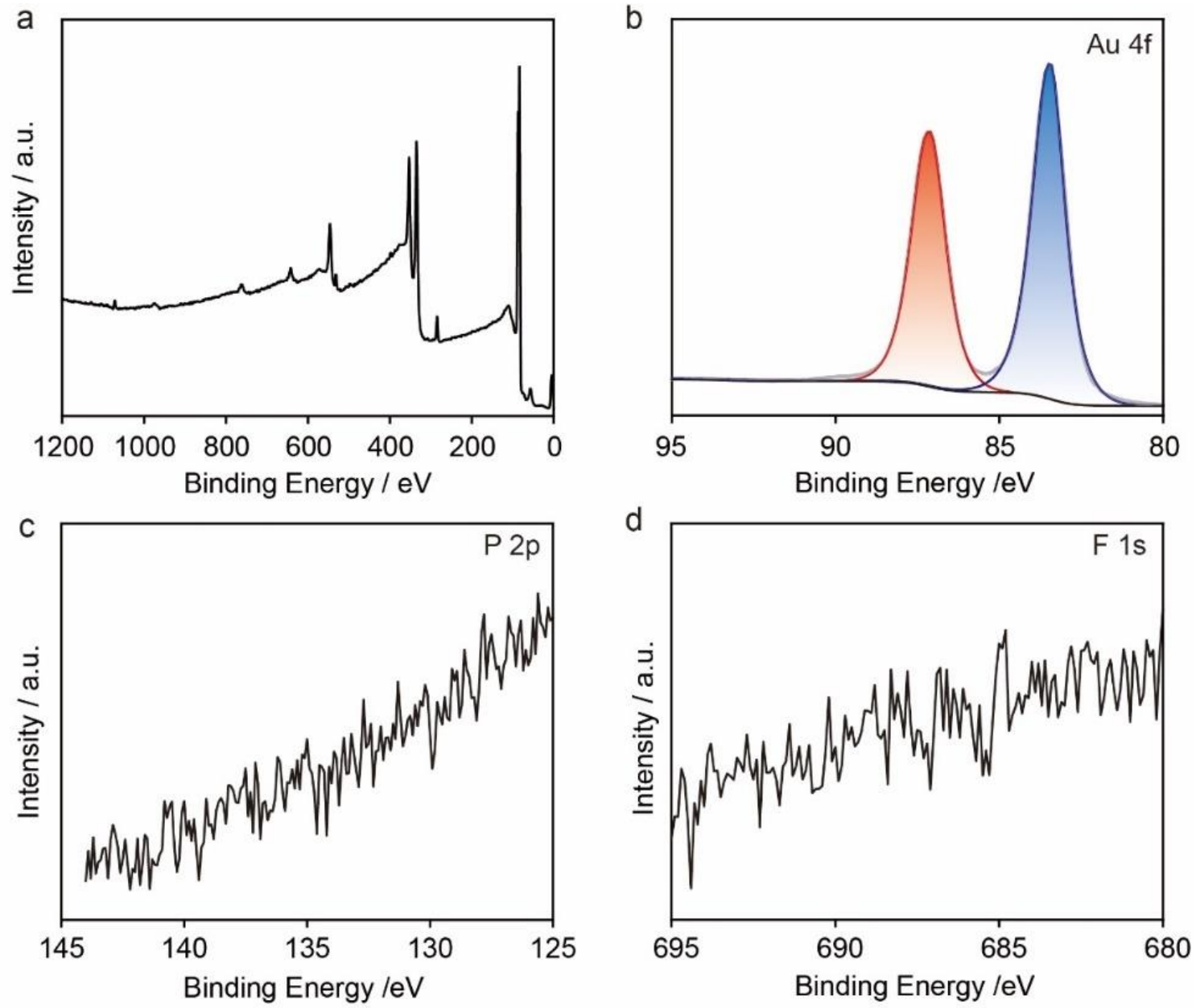

Figure S22. XPS characterizations of BIPY monolayer on Au surface. (a) XPS survey scan.

(b-d) High-resolution XPS spectrum of the (b) Au (4f), (c) P (2p), and (d) F (1s) region. 

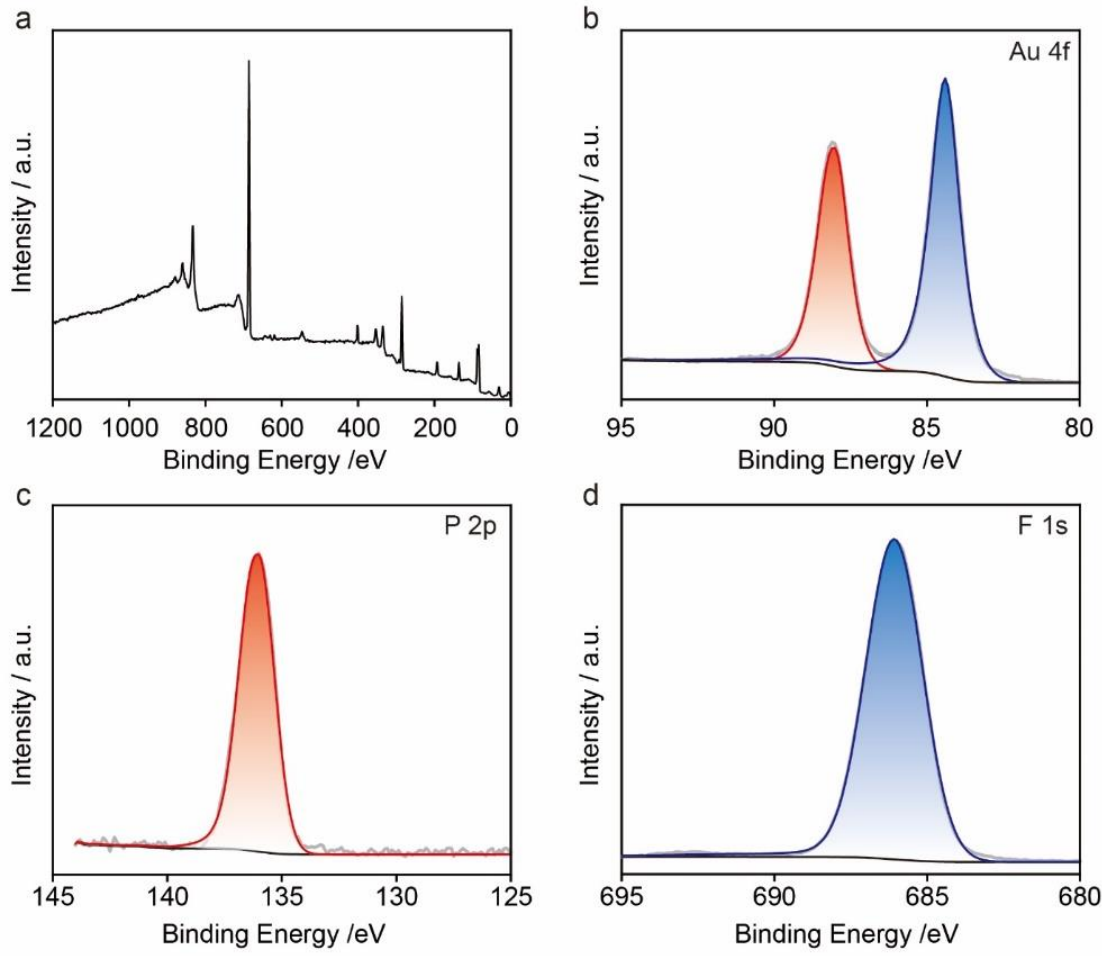

Figure S23. XPS characterizations of BIPY-Me $2 \mathrm{PF}_{6}$ monolayer on Au surface. (a) XPS survey scan. (b-d) High-resolution XPS spectrum of the (b) Au (4f), (c) P (2p), and (d) F (1s) region. The appearance of new transitions from $\mathrm{P}$ and $\mathrm{F}$ (elements in the $\mathrm{PF}_{6}{ }^{-}$counterion), compared with Figure S22, confirms the successful self-assembly of BIPY-Me•2PF 6 molecules on the Au surface.

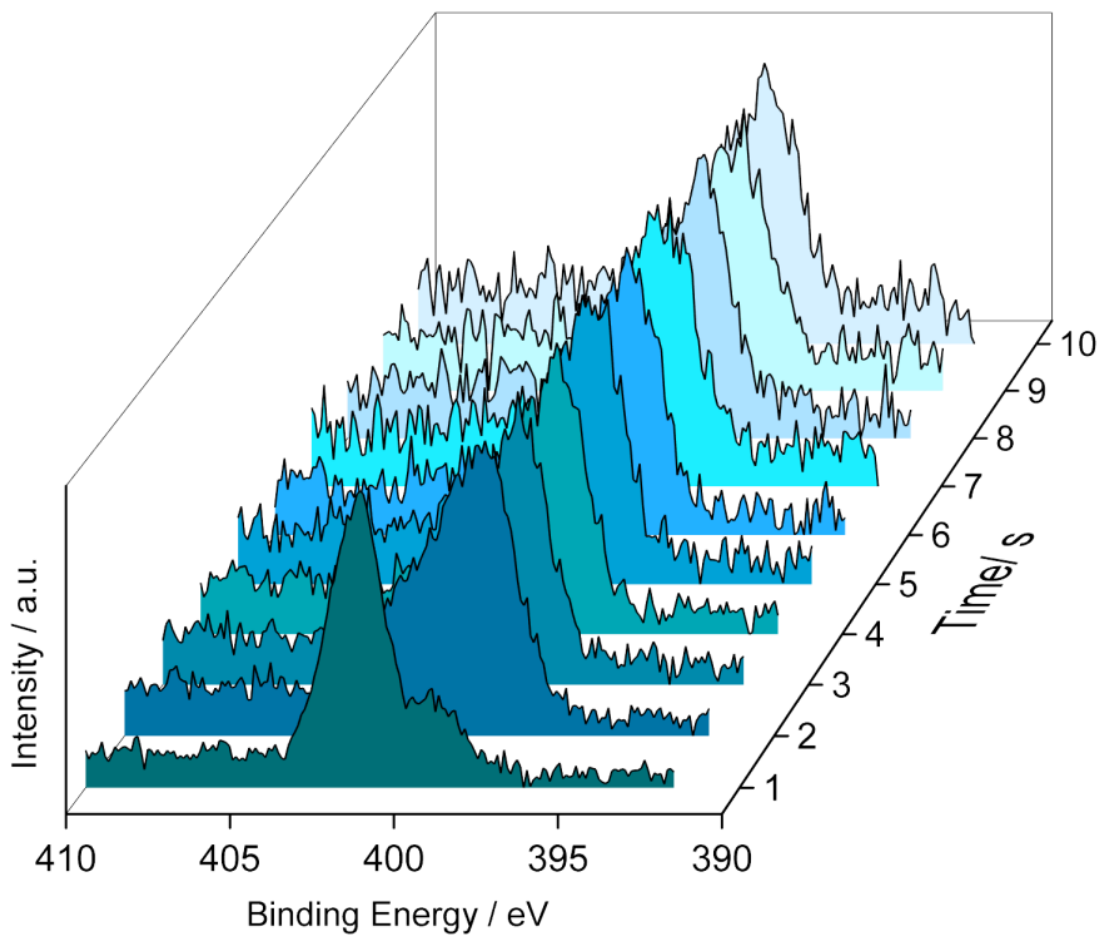

Figure S24. XPS depth analysis of BIPY-Me•2PF 6 thin film. 
After exposing to X-ray source for $1 \mathrm{~s}$, the XPS N 1s core-level spectrum gave two well-resolved peaks that correspond to viologen radical cation nitrogen (BIPY-Me ${ }^{*+}$ ) at $\sim 399.6 \mathrm{eV}$ and viologen dication nitrogen $\left(\right.$ BIPY-Me ${ }^{2+}$ ) at $\sim 401.7 \mathrm{eV}$. With prolonged X-ray exposure $(>2 \mathrm{~s})$, dication nitrogen peak decreases with the increase of the radical cation nitrogen peak, indicating that BIPY-Me ${ }^{2+}$ reduces easily to its radical state BIPY-Me ${ }^{\cdot+}$ through electron injection.

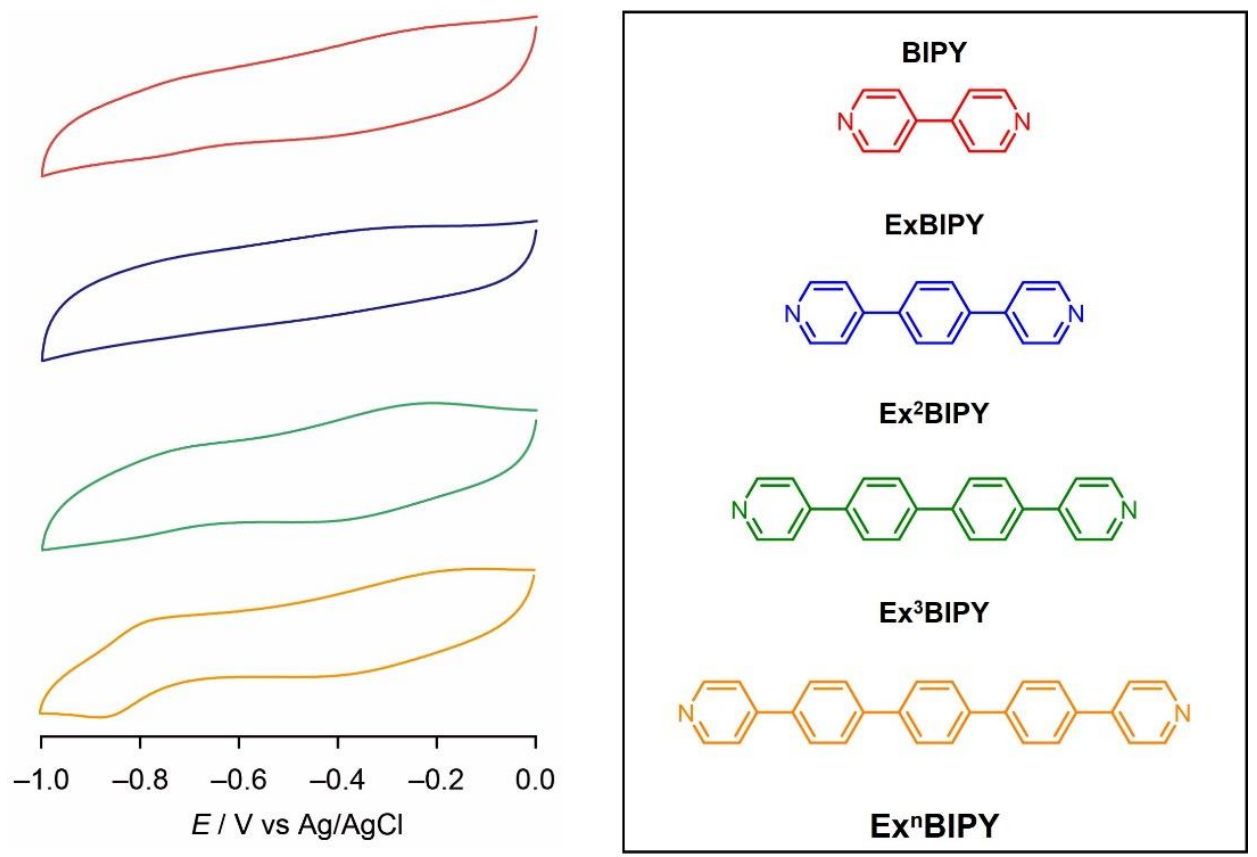

Figure S25. Cyclic voltammogram for the $\mathbf{E x}^{n} \mathbf{B I P Y}(n=0-3)$ family.
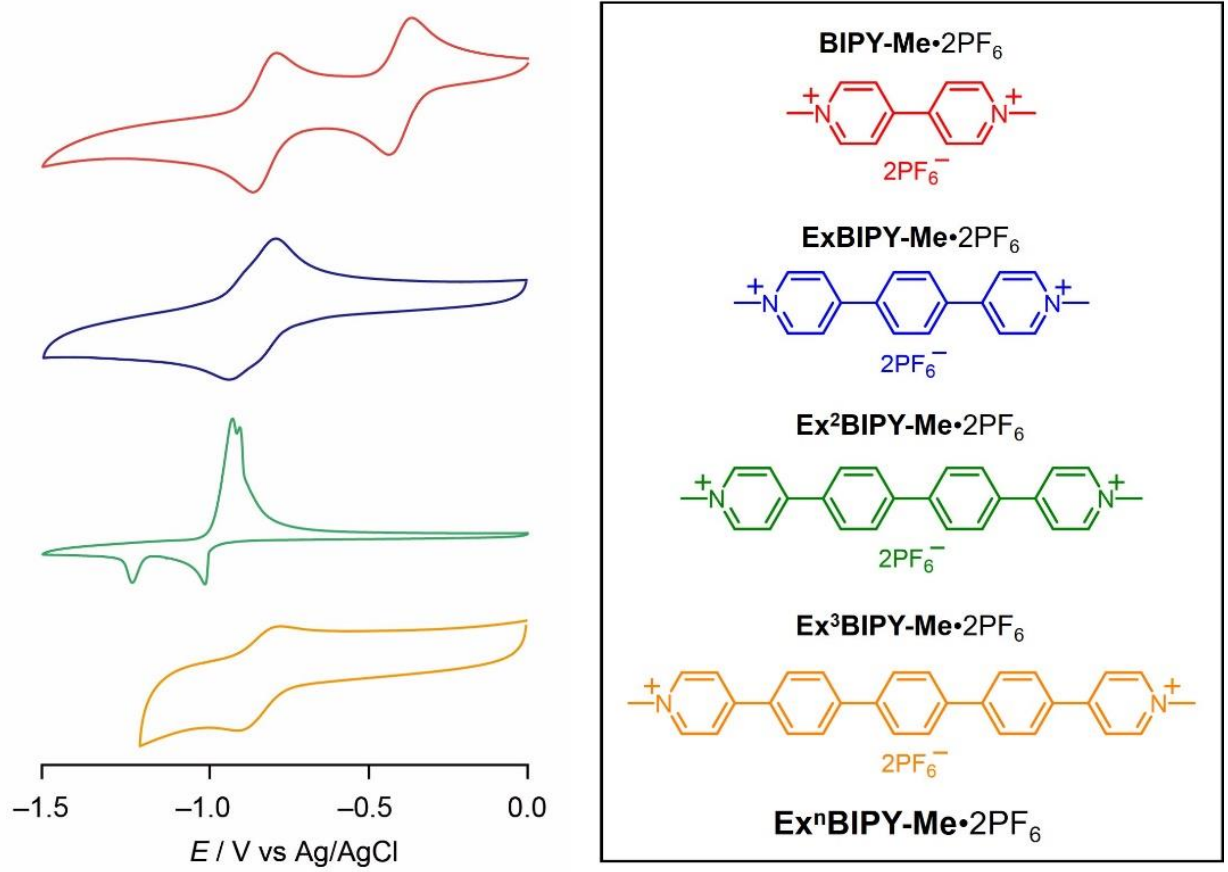

Figure S26. Cyclic voltammogram for the $\mathbf{E x}^{n} \mathbf{B I P Y}-\mathbf{M e}^{2+}(n=0-3)$ family. 


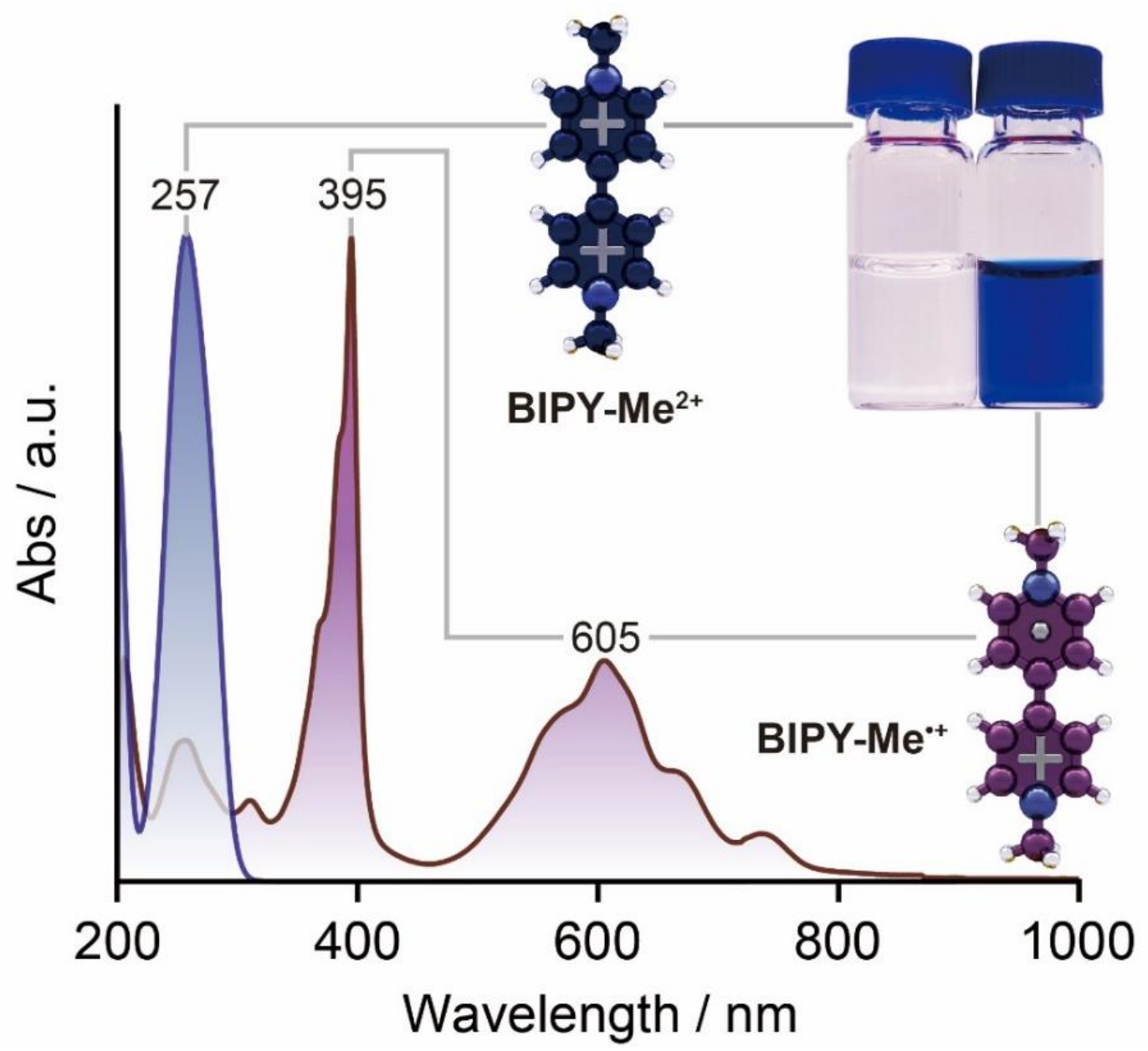

Figure S27. UV-Vis absorptions of dicationic BIPY-Me ${ }^{2+}$ and radical cationic BIPY-Me ${ }^{\bullet+}$.

The radical was generated from chemical reduction in a MeCN solution of $\mathbf{B I P Y}-\mathbf{M e}^{2+}(0.2 \mathrm{mM})$ upon addition of excess amount of Zn dust in a glovebox. All spectra were recorded with the solution filtered using $0.2 \mu \mathrm{m}$ PTFE syringe filter and sealed in a cuvette before the measurement.

\section{Section E. Calculation Methods}

\section{Charge Transport Calculations}

The spin-polarized transport results are shown in Figure S28 and Table S1. The monoradical monocationic state (BIPY-Me ${ }^{*+}$ ) with one unpaired electron has a higher conductance (HC) than the dicationic state $\left(\mathbf{B I P Y}-\mathbf{M e}^{2+}\right)$. Although these conductances vary with respect to experiment, the trend qualitatively agrees. 
a
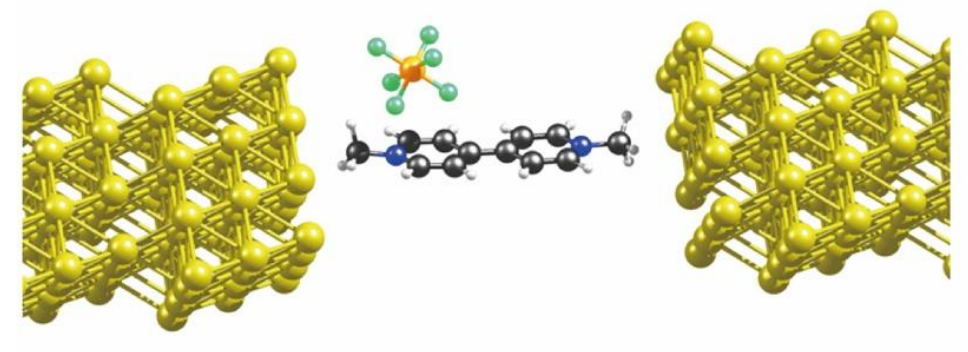

b

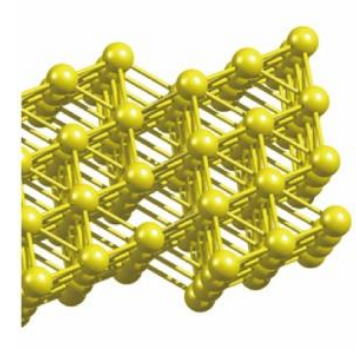

BIPY-Me ${ }^{++}$
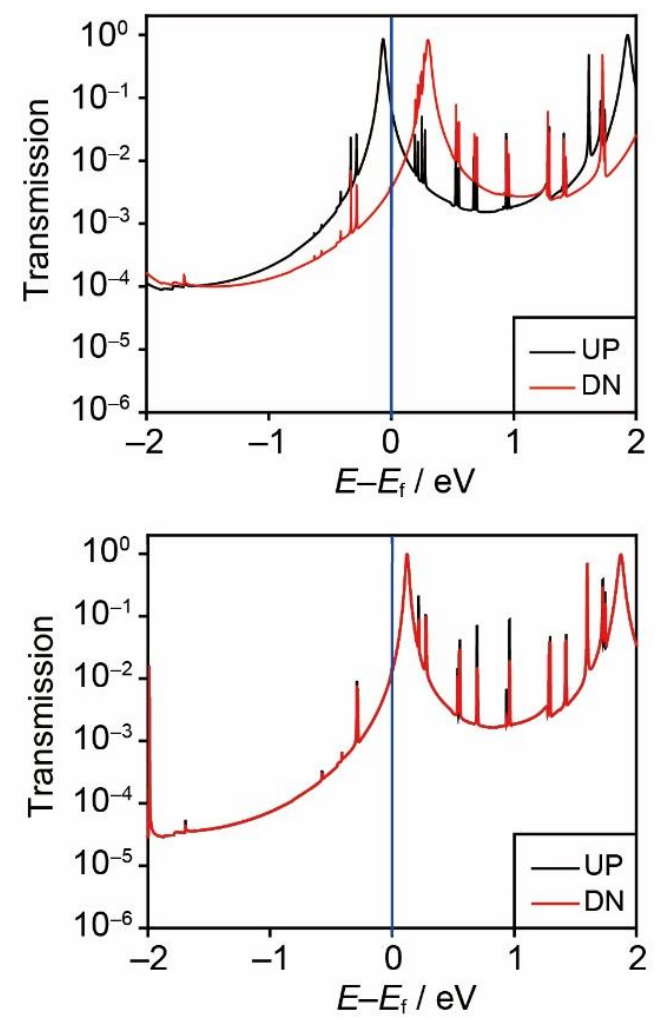

Figure S28. Molecular junctions and spin-polarized transmission spectra of (a) BIPY-Me and (b) BIPY-Me ${ }^{2+}$. The transmission spectra are with respect to the Fermi energy, on a logarithmic scale at zero bias.

Table S1. Calculated transmission function at the Fermi energy and zero bias, and conductance of BIPY-Me ${ }^{\circ+}$ and BIPY-Me ${ }^{2+}$

\begin{tabular}{ccccc}
\hline Junction & $\begin{array}{c}\text { Transmission } \\
(\mathrm{UP})\end{array}$ & $\begin{array}{c}\text { Transmission } \\
(\mathrm{DN})\end{array}$ & $\log \left(\mathrm{G}^{\left.\mathrm{UP} / \mathrm{G}_{0}\right)}\right.$ & $\log \left(\mathrm{G}^{\mathrm{DN}} / \mathrm{G}_{0}\right)$ \\
\hline BIPY-Me $^{\cdot+}$ & $7.17 \times 10^{-2}$ & $3.66 \times 10^{-3}$ & -1.15 & -2.44 \\
BIPY-Me & & & -1.89 & -1.89 \\
\hline
\end{tabular}




\section{Simulation of Electric-Field Distribution}

In order to study the electric field distribution in STM break junction quantitatively, we simulated the local electric field intensity and electric potential using the AC/DC Module (steady state) of the COMSOL Multiphysics finite-element-analysis software (COMSOL Inc). The fundamental physics equations used in this electrical field modulus includes:

$$
\begin{gathered}
J=\sigma E \\
E=\nabla V \\
\nabla J=0
\end{gathered}
$$

Where $J$ is the current density, $E$ is electrical field intensity, $V$ is electric potential, $\sigma$ is the electric conductivity. The Laplace equation is used to calculate the distribution of the electric field and electric potential:

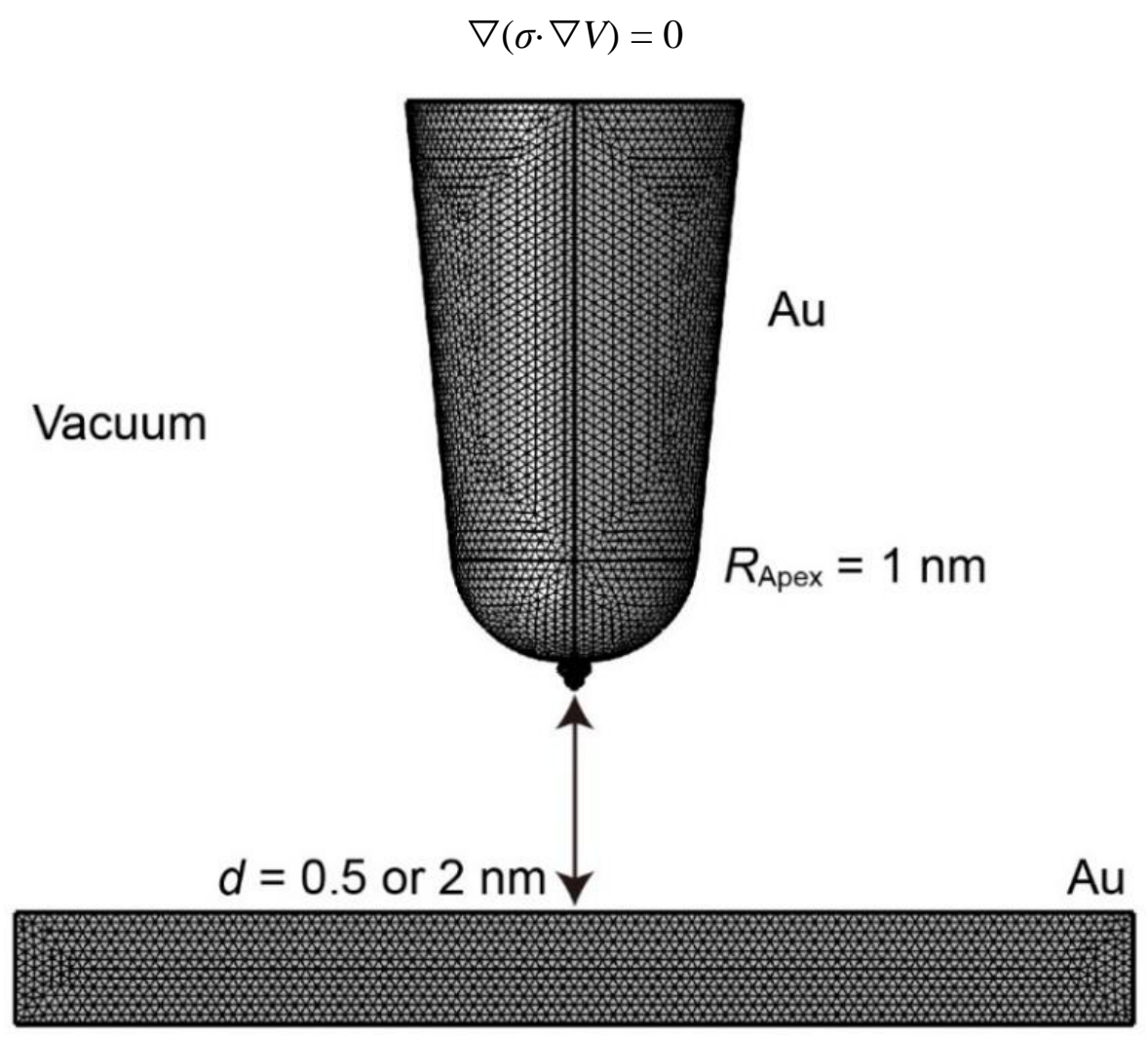

Figure S29. Schematic of the tip and sample geometry used to solve the 3D electromagnetic wave equation using FDTD numerical method. In our work, the separation $(d)$ between the $\mathrm{Au}$ tip apex and the Au substrate surface of is set to 0.5 (Figure 3c) and $2 \mathrm{~nm}$ (Figure 3d). 


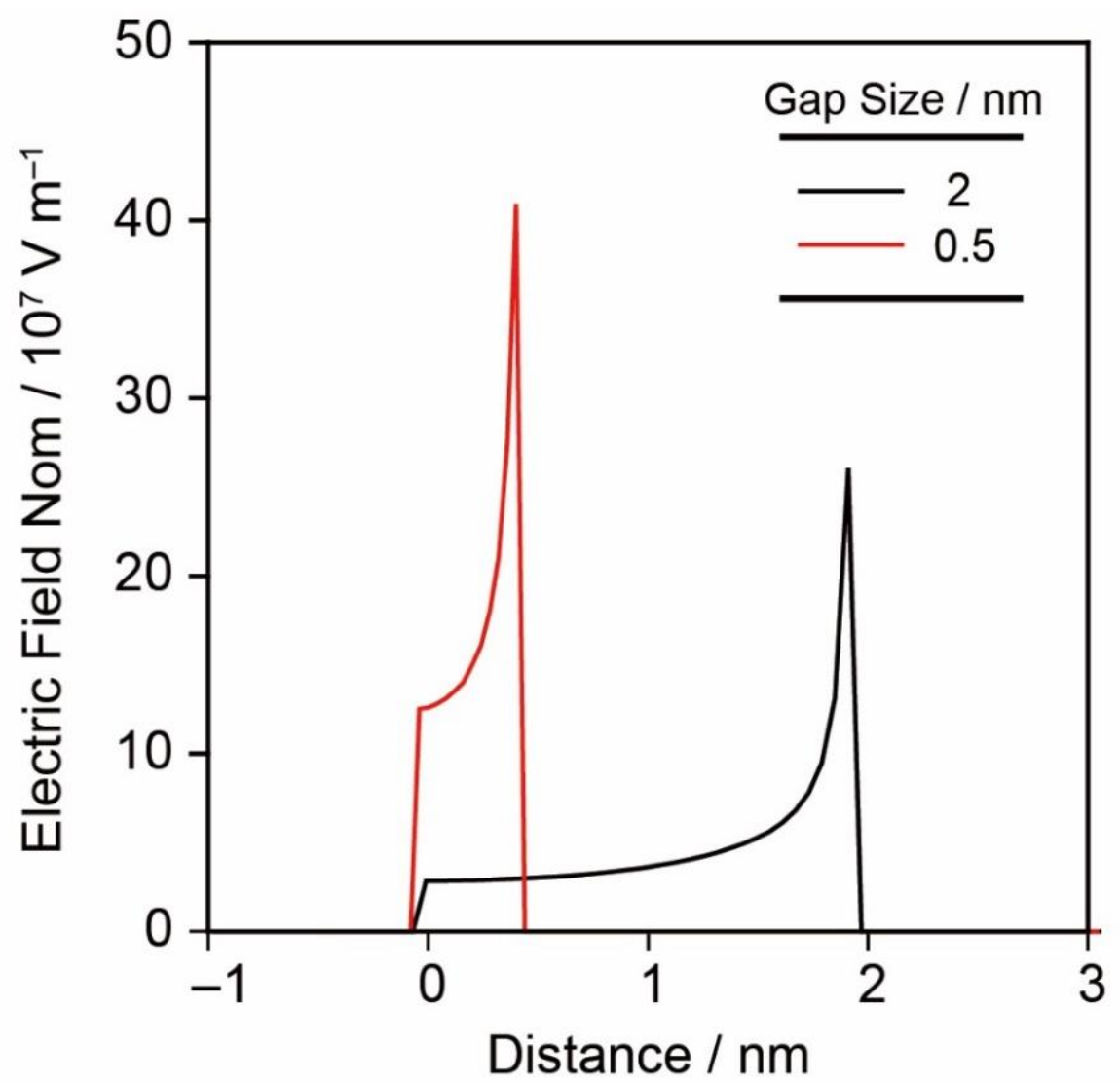

Figure S30. COMSOL simulation showing the spatial distribution of the electric field across

the break junction along the middle axis with a gap of $0.5 \mathrm{~nm}$ (red) and $2 \mathrm{~nm}$ (black) between the STM tip and the substrate.

The apex of the tip is a hemisphere with a radius of $1 \mathrm{~nm}$. The voltage $(0.1 \mathrm{~V})$ is applied between the top Au tip and the bottom Au substrate, where the top Au tip is set to be grounded. The property of the media (defined by culture medium) is considered homogeneous. The geometric center of the substrate was set as $0 \mathrm{~nm}$. The electric field in the STM junction depends largely on the gap size. In the 2-nm-size junction, the electric field in the junction is calculated to be $\sim 3.3 \times 10^{7} \mathrm{~V} \mathrm{~m}^{-1}$, and increases to a maximum of $\sim 2.6 \times 10^{8} \mathrm{~V} \mathrm{~m}^{-1}$ at the sharp end of the Au tip. In contrast, in the 0.5nm-size junction, the electric field is $\sim 2.0 \times 10^{8} \mathrm{~V} \mathrm{~m}^{-1}$ in the middle - almost one order of magnitude higher than that in 2-nm-size junction. The maximum electric field at the sharp tip end is $\sim 4.1 \times 10^{8}$ $\mathrm{V} \mathrm{m}^{-1}$ which is two times higher than that in 2-nm-size junction. 


\section{Section F. Supplemental Electrical Measurements}

STM-BJ Details. Scanning tunnelling microscope-based break junction (STM-BJ) measurements were performed using a custom-built STM that has been described ${ }^{3}$ previously. The hardware was controlled using custom software (written using LabView, National Instruments, USA) and experiments were conducted at room temperature under ambient conditions. STM Tips were prepared from electrochemically etched Au wire $(\varnothing=0.25 \mathrm{~mm}, 99.998 \%$, Alfa Aesar $)$. The Au tip was then coated ${ }^{4}$ with an insulating Apiezon wax layer to suppress background current in $\mathrm{MeCN}$. The Au substrates for solution measurements were prepared by evaporating $\mathrm{Cr} / \mathrm{Au}(10 \mathrm{~nm} / 80 \mathrm{~nm})$ at $\sim 1 \AA /$ s onto freshly cleaved mica or silicon substrates which were cleaned by Piranha solution before making measurements. The atomic contacts were freshly formed after breaking the connections between the Au tip and the substrate. Solutions $(10 \mu \mathrm{L})$ with target molecules $(0.1 \mathrm{mM})$ was dropped directly onto the Au substrate, which was used for further STM-BJ experiments.

In the case of STM-BJ measurements, the Au tip was controlled firstly by a stepper motor (Harmonic Drive, LA-30B-10-F) to obtain the approximate position to contact the substrate (less than $1 \mu \mathrm{m}$ ). Tthereafter the tip is controlled by a piezo stack (Thorlabs, PC4FL; max drive voltage, $150 \mathrm{~V}$; displacement, $4.6 \mu \mathrm{m} \pm 15 \%$ ) with the voltage applied from 0 to $10 \mathrm{~V}$, with the approach/retract at the speed of $10 \mathrm{~nm} / \mathrm{s}$. There is also a feedback system in the setup: when the voltage is changed in the piezo stack from 0 to $10 \mathrm{~V}$ one cannot contact Au tips and substrates or break the junction to the detecting limit of the amplifier (about $1 \mathrm{pA}$ ), otherwise the motor would take control of the movement of the tips to offset the distances between tips and substrates. During the repeating breaking and re-connecting operation, the bias was kept at $100 \mathrm{mV}$ and the real-time conductance was recorded using a home-built $I-V$ converter $^{5}$ with a sampling rate of $20 \mathrm{kHz}$. 
Data Analysis. Conductance was measured as a function of the tip-substrate displacement as the tip was pushed repeatedly into the substrate to reach a conductance of $>5 G_{0}$ (where $G_{0}=2 e^{2} / h, e$ is elementary charge, $h$ is Planck constant) and then retracted 5-10 nm (at $10 \mathrm{~nm} / \mathrm{s}$ ) to break the contact. In the measurement of single-molecule conductance, thousands of individual single traces are recorded in each experiment and a statistical approach without data selection is applied to determine the most probable conductance and stretching distance. All the conductance traces were used for analyses as reported in a previous paper. ${ }^{3}$ 1D-Conductance histograms were constructed by collecting all individual traces with a bin size of 1100 for $\log \left(G / G_{0}\right)$ from -10 to +1 , and 1000 for $\Delta z$ from -0.5 to $3 \mathrm{~nm}$. The conductance distribution was extracted by calculating the data density in each bin. The peak shift in a conductance histogram was determined by Gaussian fitting, which represents the most probable molecular conductance.

2D Conductance-displacement histograms were plotted by overlapping each individual trace with a bin size of 1100 for $\log \left(G / G_{0}\right)$ from -10 to +1 , and 1000 for $\Delta z$ from -0.5 to $3 \mathrm{~nm}$. All traces are aligned with a relative zero point $(\Delta z=0)$ at $G=0.5 G_{0}$. Then the $2 \mathrm{D}$ conductance distribution versus the relative distance was constructed by the data counts in each bin. In order to construct the displacement distribution histograms, firstly the relative stretching distance, $\Delta z$, was determined from the position where the conductance is $0.5 G_{0}$ (after the rupture of the gold-gold atomic break at $G_{0}$ ), to the molecular conductance region, just before the end of molecular plateau. The peak represents the most probable plateau length. In order to find the absolute displacement, $z^{*}$, which is related to the most probable length of molecular junction, the relative displacements were corrected by adding the snap-back distance, $\Delta z_{\text {corr }}$, to the relative displacement $\Delta z$, namely, $z^{*}=\Delta z$ $+\Delta z_{\text {corr. }}$ Referring to previous result, ${ }^{6} \Delta z_{\text {corr }}$ was determined experimentally to be $0.5 \pm 0.1 \mathrm{~nm}$. 

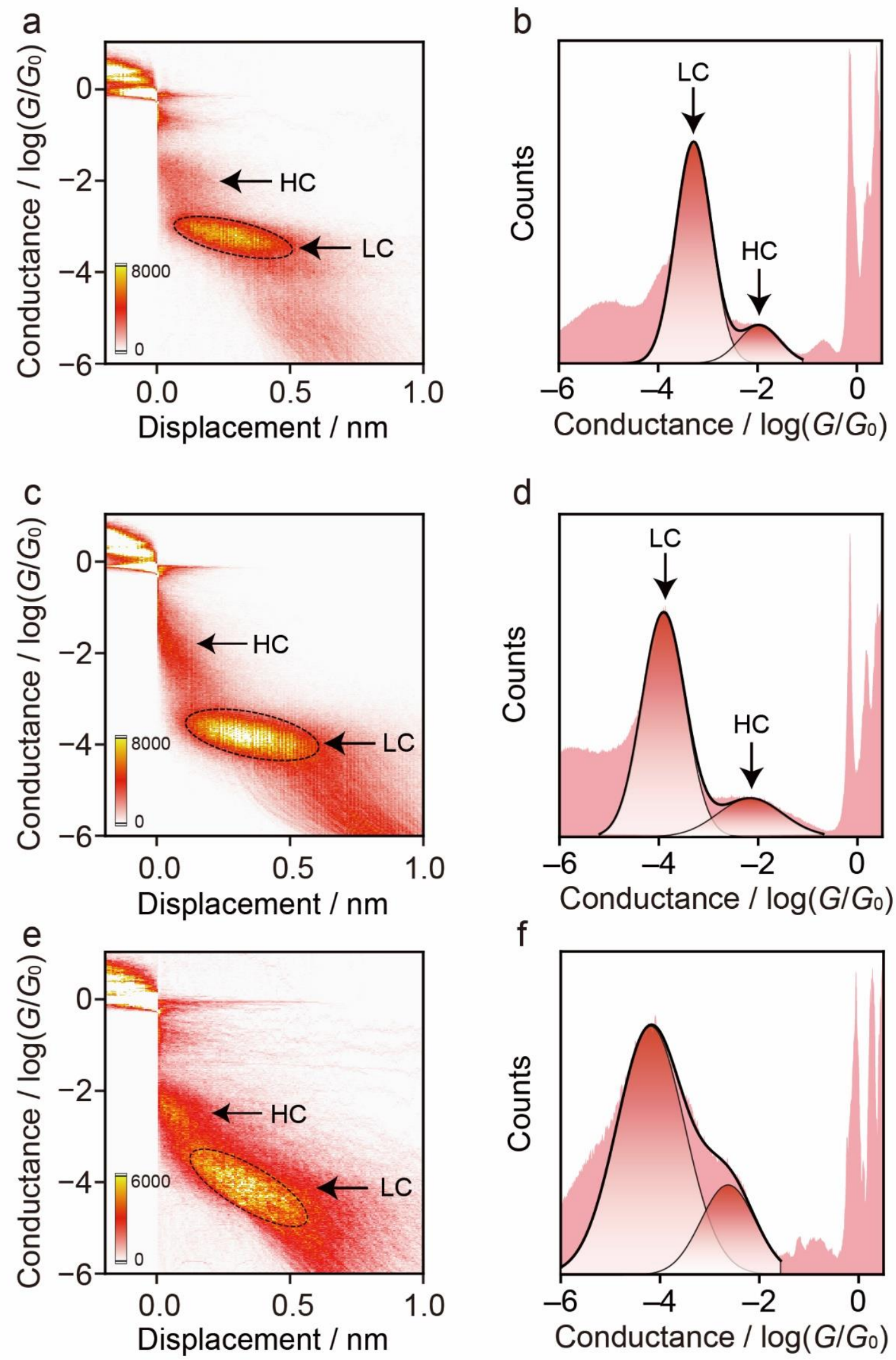

Figure S31. 2D and 1D conductance-displacement histograms for BIPY-Me $2 \mathrm{PF}_{6}$. 
a

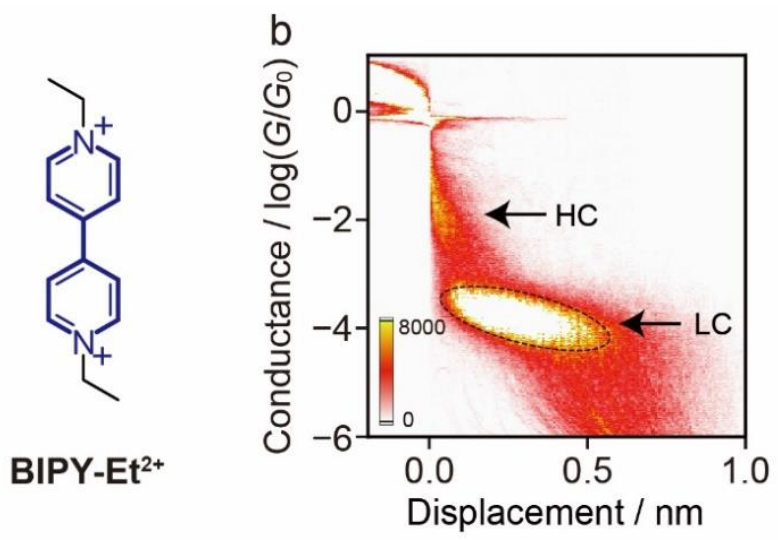

d<smiles>CCC[n+]1ccc(-c2cc[n+](CCC)cc2)cc1</smiles>

$\mathrm{BIPY}-n \mathrm{Pr}^{2+}$

g<smiles>CC(C)[n+]1ccc(-c2cc[n+](C(C)C)cc2)cc1</smiles>

BIPY-iPr ${ }^{2+}$

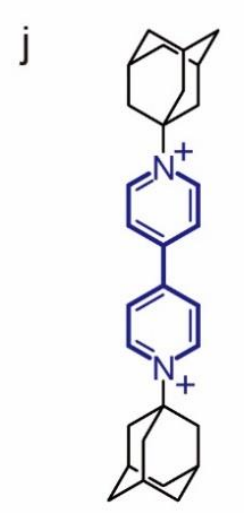

BIPY-Ada ${ }^{2+}$
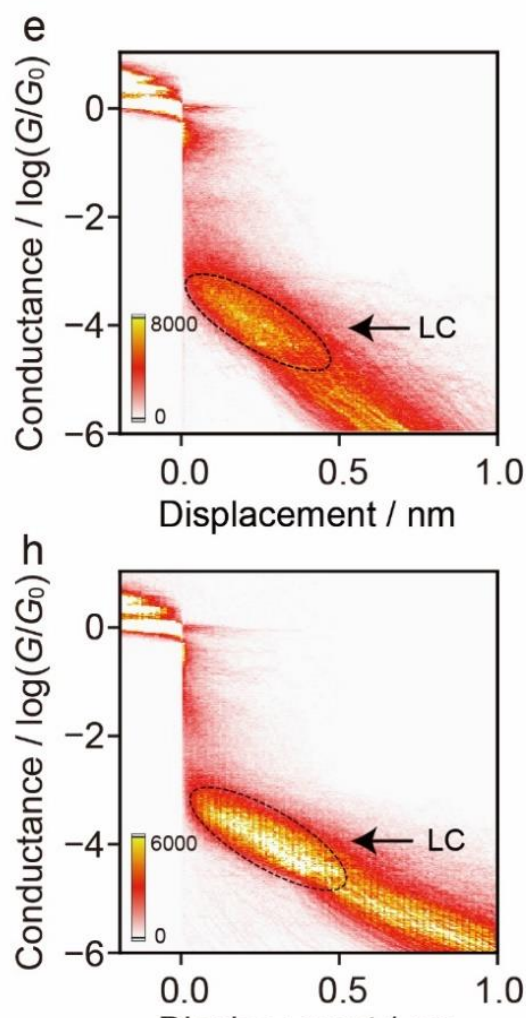

Displacement / nm

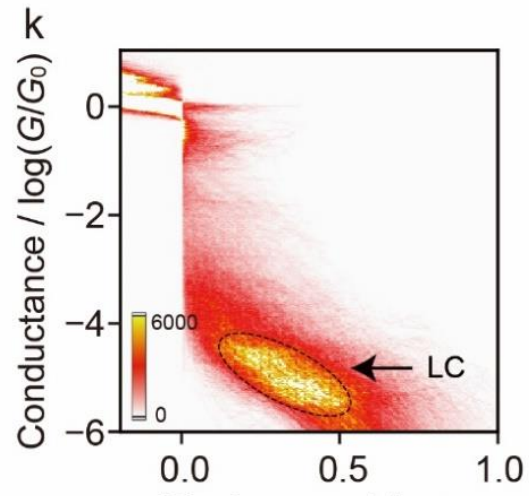

Displacement / nm
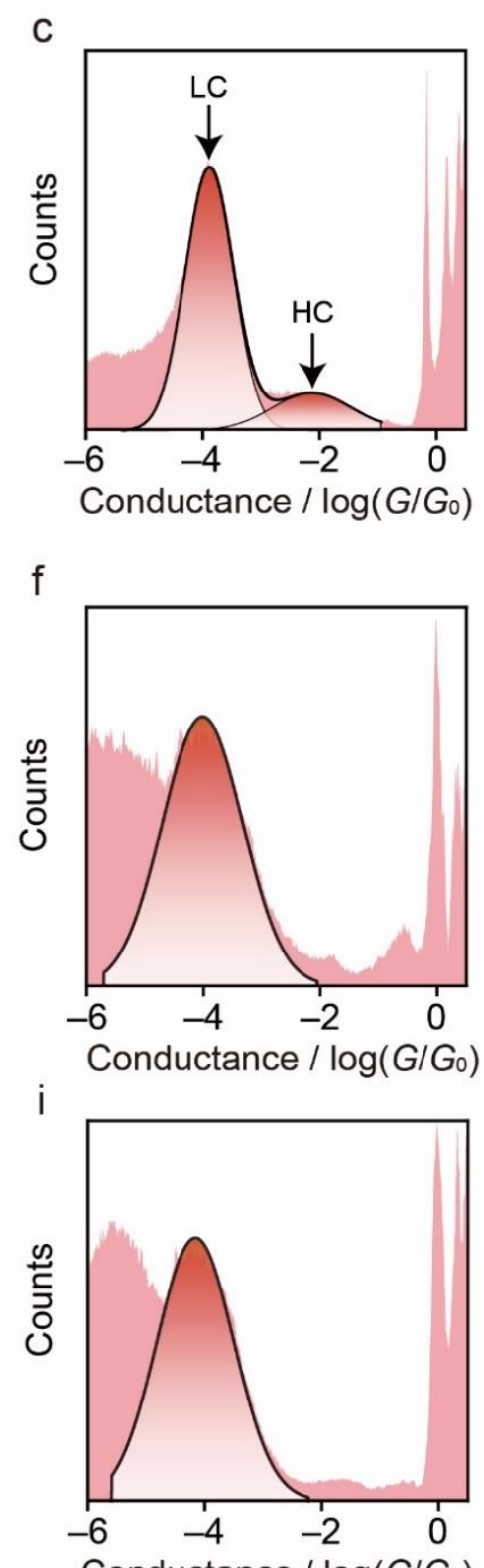

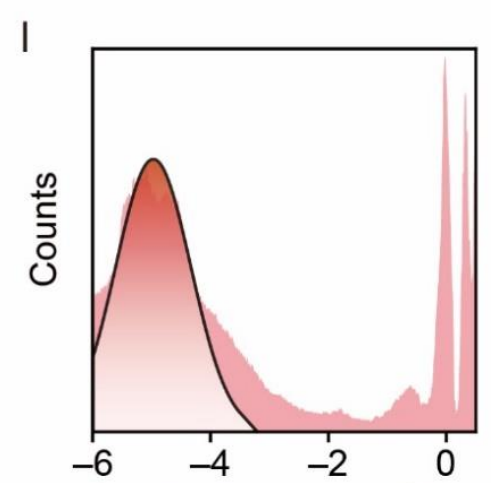

Conductance / $\log \left(G / G_{0}\right)$

Figure S32. 2D and 1D conductance-displacement histograms for BIPY-Et• $2 \mathrm{PF}_{6}(\mathrm{a}-\mathrm{c}), \mathrm{BIPY}-$ $n \mathbf{P r}^{\circ} 2 \mathrm{PF}_{6}(\mathrm{~d}-\mathrm{f}), \mathbf{B I P Y}-\boldsymbol{i P r} \cdot 2 \mathrm{PF}_{6}(\mathrm{~g}-\mathrm{i})$, and BIPY-Ada $2 \mathrm{PF}_{6}(\mathrm{j}-1)$. 

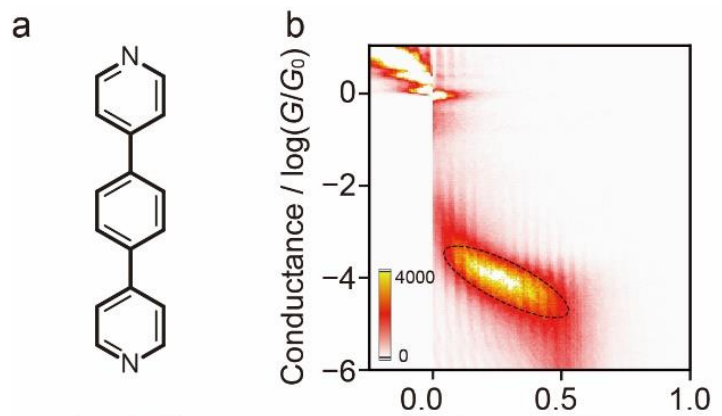

ExBIPY

d<smiles>C[n+]1ccc(-c2ccc(-c3cc[n+](C)cc3)cc2)cc1</smiles>

ExBIPY-Me ${ }^{2+}$

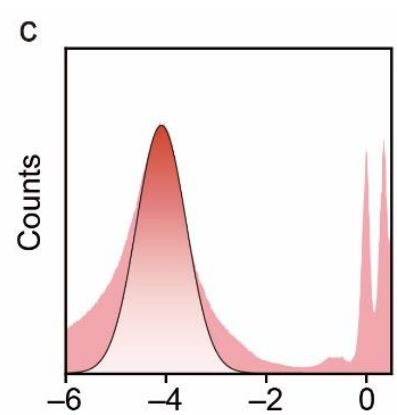

Conductance / $\log \left(G / G_{0}\right)$

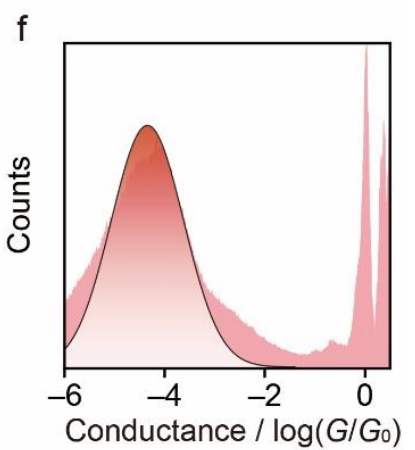

Figure S33. 2D and 1D conductance-displacement histograms for ExBIPY $(a-c)$ and ExBIPY$\mathrm{Me} \cdot 2 \mathrm{PF}_{6}(\mathrm{~d}-\mathrm{f})$.

a<smiles>c1cc(-c2ccc(-c3ccc(-c4ccncc4)cc3)cc2)ccn1</smiles>

Ex²BIPY

d<smiles>C[n+]1ccc(-c2ccc(-c3ccc(-c4cc[n+](C)cc4)cc3)cc2)cc1</smiles>

b

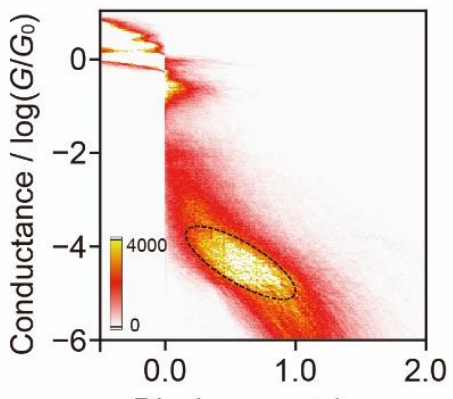

Displacement / nm

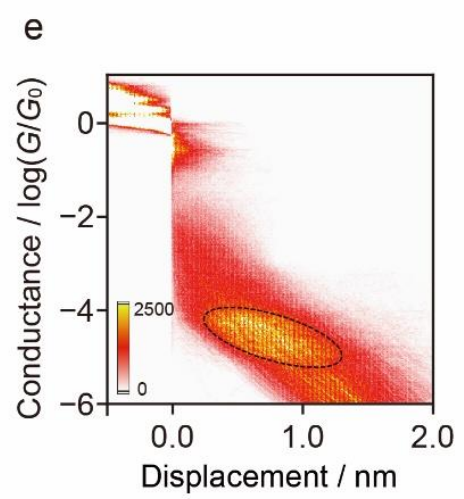

C

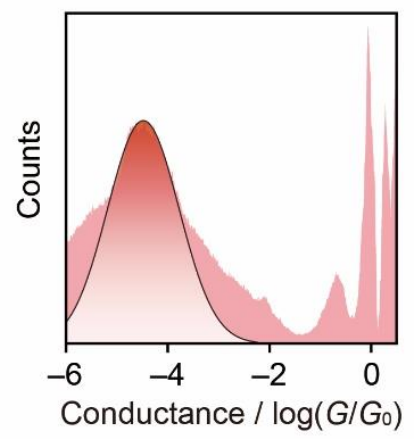

f

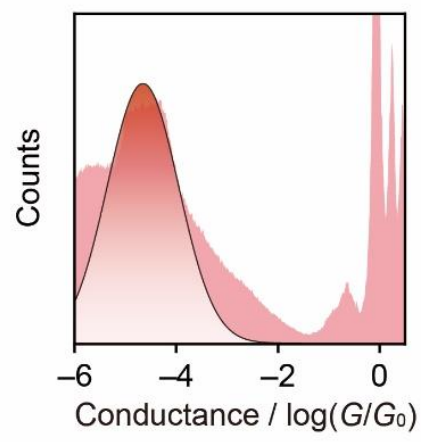

Ex²BIPY-Me ${ }^{2+}$

Figure S34. 2D and 1D conductance-displacement histograms for $\operatorname{Ex}^{2} B I P Y(a-c)$ and $\mathbf{E x}^{2} \mathbf{B I P Y}-\mathrm{Me} \cdot 2 \mathrm{PF}_{6}(\mathrm{~d}-\mathrm{f})$. 
a<smiles>CC(C)(C)[n+]1ccc(-c2ccc(-c3ccc(-c4ccc(-c5ccncc5)cc4)cc3)cc2)cc1</smiles>

d<smiles>C[n+]1ccc(-c2ccc(-c3ccc(-c4ccc(-c5cc[n+](C)cc5)cc4)cc3)cc2)cc1</smiles>

b

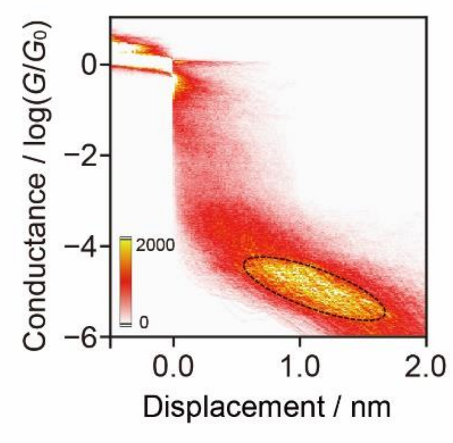

e

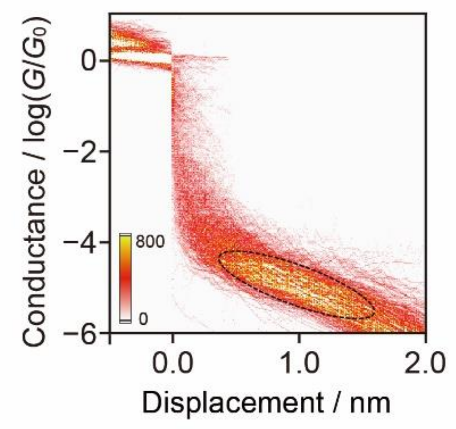

C

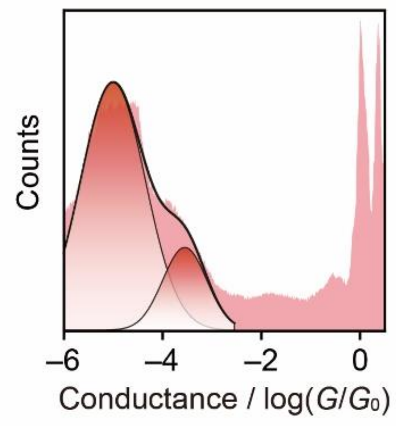

f

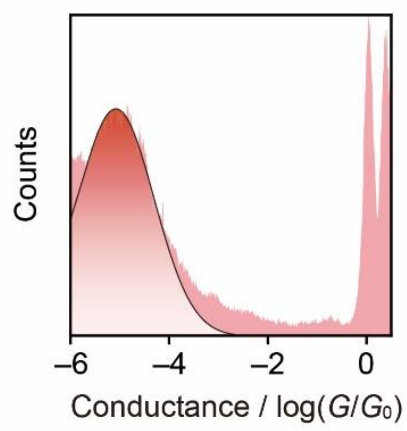

$\mathrm{Ex}^{3} \mathrm{BIPY}-\mathrm{Me}^{2+}$

Figure S35. 2D and 1D conductance-displacement histograms for $\operatorname{Ex}^{3} B I P Y(a-c)$ and $\mathbf{E x}^{3} \mathbf{B I P Y}-\mathrm{Me} \cdot 2 \mathrm{PF}_{6}(\mathrm{~d}-\mathrm{f})$.

a<smiles>C[n+]1ccc(-c2cc[n+](C)cc2)cc1</smiles>

BIPY-Me $B_{4}$

d<smiles>C[n+]1ccc(-c2cc[n+](C)cc2)cc1</smiles>

$\mathrm{BIPY}-\mathrm{Me} \cdot \mathrm{SbF}_{6}$

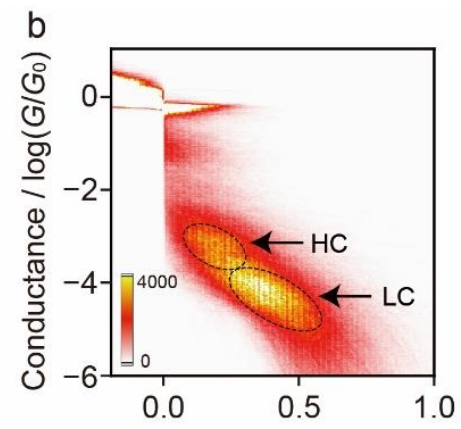

Displacement / nm

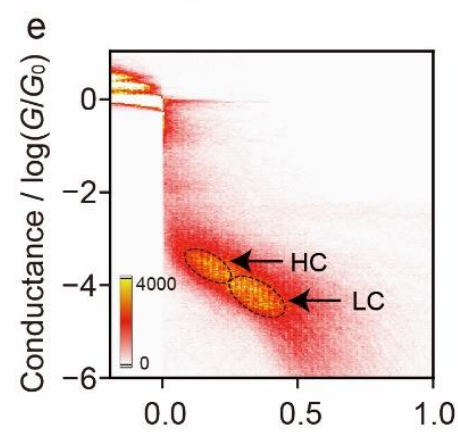

Displacement / nm

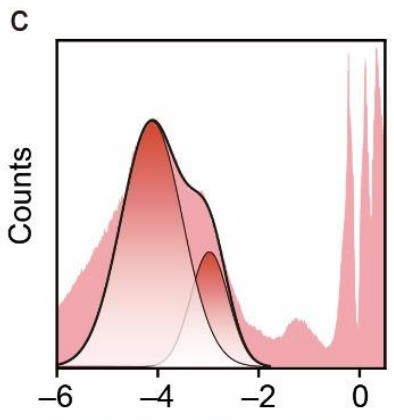

Conductance $/ \log \left(G / G_{0}\right)$

$\mathrm{f}$

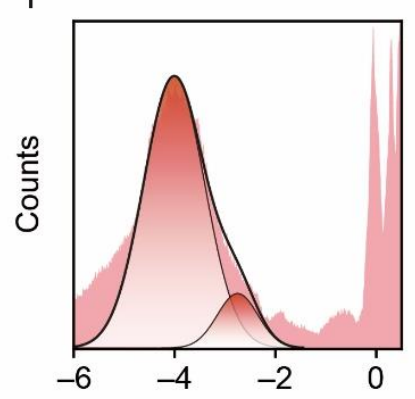

Conductance / $\log \left(G / G_{0}\right)$

Figure S36. 2D and 1D conductance-displacement histograms for BIPY-Me $2 \mathrm{BF}_{4}(\mathrm{a}-\mathrm{c})$ and BIPY-Me $2 \mathrm{SbF}_{6}(\mathrm{~d}-\mathrm{f})$. 
a BIPY-Bn·2PF 6<smiles>C[P+](C)([O+])[O-]</smiles>

C BIPY-SMeBn-2PF 6<smiles>CSc1ccc(C[n+]2ccc(-c3cc[n+](Cc4ccc(SC)cc4)cc3)cc2)cc1</smiles>

e

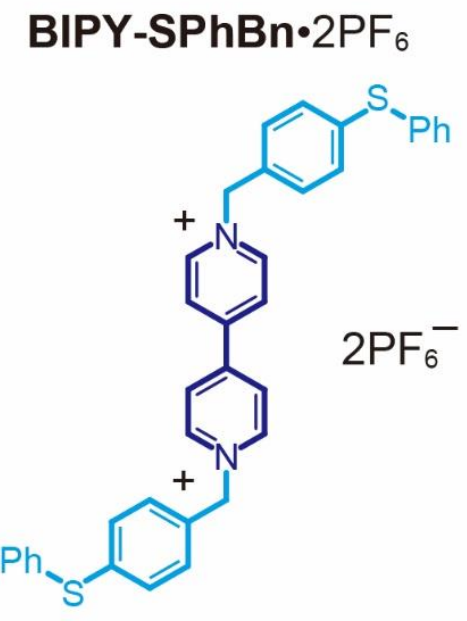

b

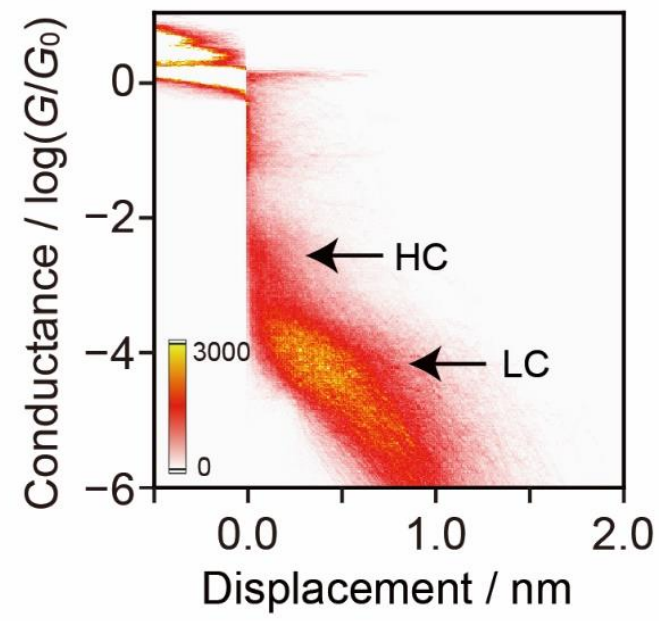

d

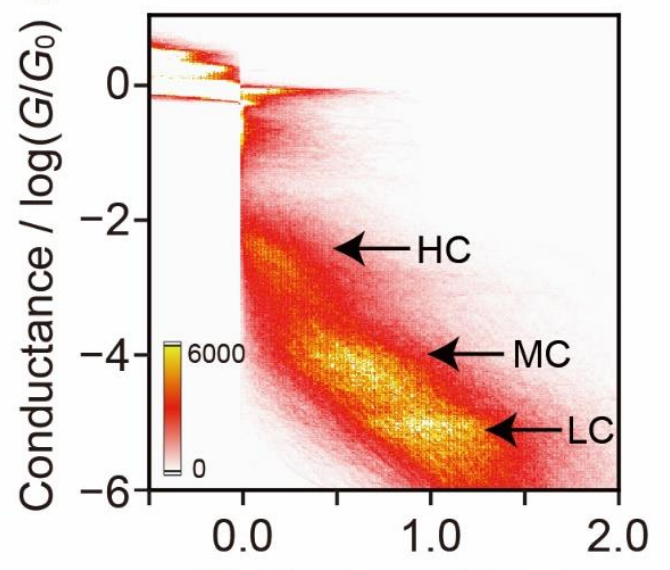

Displacement / nm

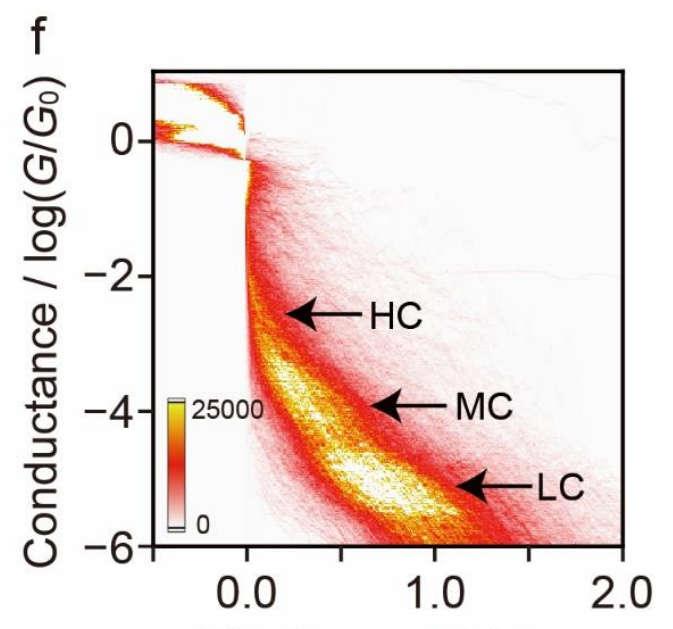

Displacement / nm

Figure S37. 2D and 1D conductance-displacement histograms for BIPY-Bn•2PF $(\mathrm{a}-\mathrm{b})$, BIPY$\mathrm{SMeBn} \cdot 2 \mathrm{PF}_{6}(\mathrm{c}-\mathrm{d})$, and BIPY-SPhBn$\cdot 2 \mathrm{PF}_{6}(\mathrm{e}-\mathrm{f})$. 


\section{Section G. Supplemental References}

1. Chen, H.; Dong, S.; Bai, M.; Cheng, N.; Wang, H.; Li, M.; Du, H.; Hu, S.; Yang, Y.; Yang, T.; Zhang, F.; Gu, L.; Meng, S.; Hou, S.; Guo, X. Solution-Processable, Low-Voltage, and HighPerformance Monolayer Field-Effect Transistors with Aqueous Stability and High Sensitivity. Adv. Mater. 2015, 27, 2113-2120.

2. Wu, H.; Chen, Y.; Zhang, L.; Anamimoghadam, O.; Shen, D.; Liu, Z.; Cai, K.; Pezzato, C.; Stern, C. L.; Liu, Y.; Stoddart, J. F. A Dynamic Tetracationic Macrocycle Exhibiting Photoswitchable Molecular Encapsulation. J. Am. Chem. Soc. 2019, 141, 1280-1289.

3. Hong, W. J.; Li, H.; Liu, S. X.; Fu, Y. C.; Li, J. F.; Kaliginedi, V.; Decurtins, S.; Wandlowski, T. Trimethylsilyl-Terminated Oligo(phenylene ethynylene)s: An Approach to Single-Molecule Junctions with Covalent Au-C sigma-Bonds. J. Am. Chem. Soc. 2012, 134, 19425-19431.

4. Nagahara, L. A.; Thundat, T.; Lindsay, S. M. Preparation and Characterization of STM Tips for Electrochemical Studies. Rev. Sci. Instrum. 1989, 60, 3128-3130.

5. Meszaros, G.; Li, C.; Pobelov, I.; Wandlowski, T. Current Measurements in a Wide Dynamic Range - Applications in Electrochemical Nanotechnology. Nanotechnology 2007, 18, 424004.

6. Manrique, D. Z.; Huang, C.; Baghernejad, M.; Zhao, X. T.; Al-Owaedi, O. A.; Sadeghi, H.; Kaliginedi, V.; Hong, W. J.; Gulcur, M.; Wandlowski, T.; Bryce, M. R.; Lambert, C. J. A Quantum Circuit Rule for Interference Effects in Single-Molecule Electrical Junctions. Nat. Commun. 2015, $6,6389$. 